\title{
Mapping excessive "disgust" in the brain: Ventral pallidum inactivation recruits distributed circuitry to make sweetness "disgusting"
}

\author{
Hammad A. Khan ${ }^{1,2} \cdot$ Kevin R. Urstadt $^{1,3} \cdot$ Nina A. Mostovoi ${ }^{1} \cdot$ Kent C. Berridge $^{1}$ \\ Published online: 13 December 2019 \\ (C) The Psychonomic Society, Inc. 2019
}

\begin{abstract}
The ventral pallidum (VP) is an important structure in processing reward. The VP may be the only brain structure where localized lesions in rats replace normal facial "liking" expressions to sweetness with excessive "disgust" reactions, such as gapes and chin rubs, that are normally reserved for unpalatable tastes. The posterior half of the VP ( $\mathrm{pVP}$ ) contains a hedonic hot spot where opioid or related neurochemical stimulations can amplify positive "liking" reactions to sweet taste. This is the same site where lesions or pharmacological inactivations replace positive hedonic reactions to sucrose with intense negative "disgust." In the present study, we aimed to identify brain networks recruited by pVP inactivation to generate excessive "disgust," using neuronal Fos expression as a marker of neurobiological activation. Microinjections in pVP of inhibitory GABA $\mathrm{A}_{\mathrm{B}}$ agonists (muscimol and baclofen) caused rats to exhibit excessive "disgust" reactions to sucrose. Excessive "disgust" was accompanied by recruitment of neural Fos activation in several subcortical structures, including the posterior medial shell of nucleus accumbens (which also contains another GABAergic "disgust"-inducing "hedonic cold spot"), the bed nucleus of stria terminalis, lateral habenula, hypothalamus, and midbrain ventral tegmentum. Fos suppression was found in cortical limbic regions, including previously identified hedonic hot spots in the anteromedial orbitofrontal cortex and posterior insula. Finally, in addition to inducing excessive "disgust," pVP inactivation abolished motivational "wanting" to eat palatable food, reduced positive social interactions, and reordered sensorimotor relations. Our findings identify potential "disgust" generators in the brain that are released into excitation by pVP inhibition and may serve as targets for future research.
\end{abstract}

Keywords Animal models $\cdot$ Reward $\cdot$ Emotion $\cdot$ Motivation $\cdot$ Neural network

Positive hedonic reactions of "liking" for pleasant stimuli are crucial for normal psychological function and mental wellbeing. Affective dysfunction in hedonic brain mechanisms involving excessive negative affect characterizes various clinical disorders, including depression, anxiety, anorexia nervosa, borderline personality disorder, obsessive-

Hammad A. Khan

hak53@case.edu

1 Department of Psychology, University of Michigan, Ann Arbor, MI, USA

2 Case Western Reserve University School of Medicine, Health Education Campus, 9501 Euclid Avenue, Cleveland, OH 44106, USA

3 Department of Cognitive Science, Occidental College, Los Angeles, CA, USA compulsive disorder, and phobias (Arrondo et al., 2015; reviewed in Davey, 2011).

Disgust is a prototypical form of negative affect, and in its most basic form, prototypical sensory "disgust" expressions (e.g., gapes) are elicited by bitterness and other unpalatable tastes in human infants, other primates, and rats (Berridge, 2000; Darwin, 1872; Grill \& Norgren, 1978a; Keltner \& Ekman, 2000; Pfaffman, Norgren, \& Grill, 1977; Rozin, Haidt, \& McCauley, 2008; Steiner, 1973; Steiner, Glaser, Hawilo, \& Berridge, 2001). In contrast to negatively valenced "disgust" reactions, positive "liking" facial expressions are normally elicited in infants and animals by sweetness and other preferred tastes (e.g., lip licking). Quotation marks around "liking" and "disgust" are used here to distinguish such core affective reactions, which can be objectively measured, from conscious subjective feelings of liking and disgust that may accompany them and are denoted without quotation marks (Berridge, 2018). 
Brain manipulations that release "disgust" reactions may be used to help reveal the causal brain mechanisms that generate excessive "disgust," such as after dysfunction in the pVP or related limbic circuitry (Cromwell \& Berridge, 1993, 1994; Faure, Richard, \& Berridge, 2010; Ho \& Berridge, 2014; Morgane, 1961; Reynolds \& Berridge, 2002; Schallert \& Whishaw, 1978; Teitelbaum \& Epstein, 1962). A better understanding of brain networks that generate "disgust" and related affective reactions may help research and improve treatments for related clinical disorders.

The ventral pallidum (VP) is an important component of brain reward and pleasure circuitry (Smith, Tindell, Aldridge, \& Berridge, 2009). The posterior half of the VP (pVP), in particular, contains a $0.8 \mathrm{~mm}^{3}$ "hedonic hot spot," in which opioid or orexin neurochemical stimulation by microinjection can nearly double positive orofacial "liking" reactions to the hedonic impact of sweet tastes (Ho \& Berridge, 2013; Smith $\&$ Berridge, 2005, 2007). The $\mathrm{pVP}$ hot spot is one of several hedonic hot spots distributed across the brain, including in nucleus accumbens (NAc), orbitofrontal cortex (OFC), posterior insula, and brain stem, which together form an interacting hedonic network for amplification of "liking" (Berridge \& Kringelbach, 2015; Castro \& Berridge, 2017; Peciña \& Berridge, 2005). Neuronal firing in the pVP hot spot tracks the magnitude of positive "liking" reactions to different tastes, and similarly tracks changes in "liking" reactions to the same sweet or salty taste induced by homeostatic appetite/satiety shifts or by opioid microinjection stimulation in another hedonic hot spot (Smith, Berridge, \& Aldridge, 2011; Tindell, Berridge, \& Aldridge, 2004; Tindell, Smith, Peciña, Berridge, \& Aldridge, 2006)

The $\mathrm{pVP}$ is unique among these hedonic hot spots, however, in that it is the only brain site where small focused lesions or neuronal loss is known to eliminate normal "liking" reactions to a normally pleasant stimulus, such as sucrose taste, and replace them with excessive "disgust" (Cromwell \& Berridge, 1993; Ho \& Berridge, 2014). Excessive "disgust" was produced in early studies by large electrolytic lesions (which destroy both neurons and axonal fibers of passage) of the greater lateral hypothalamus region that included the ventral pallidum, and in later studies by excitotoxic lesions (which destroy only local neurons) that either damaged the entirety of the hypothalamic-pallidal region or more specifically damaged only the $\mathrm{pVP}$. By contrast, lesions of the lateral hypothalamus specifically do not increase "disgust" as long as the pVP site remains intact (Cromwell \& Berridge, 1993, 1994; Morgane, 1961; Schallert \& Whishaw, 1978; Teitelbaum \& Epstein, 1962). This anatomical pattern underscores the special role of the pVP in releasing "disgust" in response to neuronal damage. Beyond lesions, excessive "disgust" reactions to sucrose taste is also produced by temporary inactivation of $\mathrm{pVP}$ neurons, via microinjections of $\mathrm{GABA}_{\mathrm{A} / \mathrm{B}}$ agonists such as muscimol and baclofen, either in the $\mathrm{pVP}$ hot spot (Ho \& Berridge, 2014; Shimura, Imaoka, \& Yamamoto, 2006) or in a hedonic "cold spot" located in the posterior half of NAc medial shell (where even opioid microinjections suppress "liking"; Faure et al., 2010; Ho \& Berridge, 2014; Reynolds \& Berridge, 2002).

Pharmacologically, microinjections of the $\mathrm{GABA}_{\mathrm{A} / \mathrm{B}}$ agonists muscimol and baclofen in the pVP induce excessive "disgust" by binding GABA receptors and causing them to open chloride ion channels. The resulting influx of negatively charged chloride ions hyperpolarizes neurons in the $\mathrm{pVP}$, inhibiting their firing and reducing neurotransmitter release. We hypothesize that this inhibition of pVP hot spot neurons removes its crucial contributions to positive hedonic brain function, causing loss of normal "liking" reactions to sweetness. This has been further hypothesized to release negatively valenced "disgust"-generating neural circuitry elsewhere in the brain, which consequently becomes free to generate and express excessive levels of negatively valenced "disgust" (Ho \& Berridge, 2014). However, the identity of this released "disgust"-generating neural circuitry is as yet unknown. As a step toward identifying "disgust"-generating circuitry, we aimed here to map brain structures that become neurobiologically activated following pVP inactivation that causes excessive "disgust." Specifically, we mapped patterns of changed neuronal Fos expression throughout several limbic structures in the brain, to identify where neuronal transcription and translation of the early immediate gene c-fos into the Fos protein became elevated after $\mathrm{pVP}$ inactivation that induced sweetness "disgust."

\section{Material and methods}

Inactivation of the $\mathrm{pVP}$ was accomplished by bilateral microinjections of muscimol + baclofen aimed at the pVP hot spot. After confirming excessive "disgust" reactions to sucrose taste, we examined Fos expression in subregions of a number of limbic structures: hypothalamus, thalamus and lateral habenula, limbic cortex (orbitofrontal, cingulate, and insula regions), neostriatum, mesolimbic nucleus accumbens, ventral tegmentum, substantia nigra, subthalamic nucleus, bed nucleus of the stria terminalis, brain stem nucleus of the solitary tract, and parabrachial nucleus of the pons. Prior to that final test, we also assessed the effects of pVP inactivation on food intake and on social interaction and social avoidance to compare induced "disgust" to other forms of negative affect.

\section{Subjects}

Sprague Dawley rats $(N=42$ total; 18 male, 24 female) weighing $250-450 \mathrm{~g}$ at the time of surgery were housed in same-sex pairs or triplets at $\sim 21^{\circ} \mathrm{C}$ on a reverse 12 -hr light/ dark cycle (behavioral testing occurred during the rats' 
behaviorally active dark cycle). Rats were handled and acclimated prior to the experimental procedures below. All rats had ad libitum access to food (standard Purina Rat Chow) and tap water in their home cages throughout the study, with the exception of 10 rats (four male, six female) who were food restricted for $24 \mathrm{hr}$ before two of four testing sessions, as described below. All experimental procedures were conducted in accordance with protocol approved by the Institutional Animal Care and Use Committee (IACUC) at the University of Michigan.

\section{Surgery}

Rats were anesthetized with ketamine hydrochloride (80 $\mathrm{mg} / \mathrm{kg}$, IP) and xylazine ( $5 \mathrm{mg} / \mathrm{kg}$ IP), pretreated with atropine $(0.05 \mathrm{mg} / \mathrm{kg}$, IP) to prevent respiratory distress, and placed in a stereotaxic apparatus (David Kopf Instruments) with the incisor bar set to flat (3.3 mm below the interaural line). Rats were also pretreated with carprofen $(5 \mathrm{mg} / \mathrm{kg}, \mathrm{SQ})$ and cefazolin $(60 \mathrm{mg} / \mathrm{kg}, \mathrm{SQ})$ to reduce pain and avert infection. Bilateral intracranial guide cannulae were implanted to permit microinjections into the pVP (14 mm long, 23-gauge stainless steel tubing). Cannula tips were aimed $2 \mathrm{~mm}$ above the pVP using the following coordinates: $-0.7 \mathrm{~mm}$ posterior to breg$\mathrm{ma}, \pm 2.8 \mathrm{~mm}$ lateral to bregma, and $-6.0 \mathrm{~mm}$ ventral to the skull surface. In the same surgery, intraoral cannulae (polyethylene PE-100 tubing) were also bilaterally implanted, entering the mouth lateral to the maxillary molar on both sides, to permit oral infusions of solution during taste reactivity testing, as described in previous work (Grill \& Norgren, 1978a). Intracranial and intraoral cannulae exited at the dorsal skull and were anchored with surgical screws and dental acrylic cement. Stainless steel stylets were inserted into intracranial cannulae to avoid occlusion.

Rats received anesthetic antagonist atipamezole $(1 \mathrm{mg} / \mathrm{kg}$, SQ) immediately after surgery, received an additional dose of carprofen $24 \mathrm{hr}$ after surgery, and were given 1 week to recover following surgery. Rats were given fresh moistened cereal mash each day (Gerber's Mixed Grain Baby Cereal, combined with water) in addition to chow pellets and water for 3 days following surgery to allow for easier food consumption, and were monitored following surgery to ensure that intraoral cannulas did not disrupt normal eating or behavior. Another group of rats ( $n=6$, two male, four female) was anesthetized with isoflurane (5\% mixed with air for induction, $1 \%-2 \%$ for maintenance) and received only pVP microinjection cannulae, without intraoral cannulae, aimed at the coordinates described above.

\section{Drugs and VP microinjections}

To make bilateral pVP microinjections, rats were gently cradled in the experimenter's lap while stylets were removed, and intracranial microinjectors were inserted into guide cannulae. PE-20 polyethylene delivery tubing from a syringe pump connected to a 28 -gauge stainless steel microinjector, extending either 14 or $16 \mathrm{~mm}$ from the plastic guard, was inserted into a guide cannula. The short 14-mm microinjectors were used for "mock" injections during initial habituation sessions, while 16-mm microinjectors, which extended $2 \mathrm{~mm}$ below the tip of guide cannulas, were used for drug administration prior to behavioral test sessions. Prior to test sessions, rats received bilateral microinjections either of a muscimol-baclofen cocktail (muscimol + baclofen; $0.1 \mu \mathrm{g}$ muscimol and $0.1 \mu \mathrm{g}$ baclofen per $0.3 \mu \mathrm{L}$ artificial cerebrospinal fluid, per hemisphere) to inhibit the VP, or of artificial cerebrospinal fluid alone as a vehicle control condition. Microinjections were infused over a 1-min period, and microinjectors were subsequently left in place for $1 \mathrm{~min}$ to allow for diffusion of the drug into brain tissue, after which microinjectors were removed and stylets replaced in the guide cannula. The rat's oral cannulae were connected to intraoral delivery tubes, and the rat was gently placed in a taste reactivity chamber.

\section{Habituation and experiment schedule}

Prior to tests, rats were habituated to taste reactivity chambers and to food intake chambers for 30-min periods on 4 consecutive days, and oral cannulae were flushed with a small infusion of distilled water prior to each habituation session. All rats, except those intended for the hunger-satiety comparison, were arbitrarily assigned to either a muscimol + baclofen inactivation group ( $n=16$, eight male, eight female) or to a vehicle-only control group $(n=10$, four male, six female) for between-subject comparisons. Other rats intended for the hunger-satiety comparison ( $n=10$, four male, six female) were kept aside and treated as described below. The following sections describe the experimental time line, which is also depicted in Fig. 1.

\section{Taste reactivity after VP inactivation in ad libitum condition}

Rats that had ad libitum access to food in their home cage ( $n=$ 26 , twelve male, fourteen female) were tested for sucrose taste reactivity after receiving sham microinjections on the first test day, vehicle microinjections on the second day, and then either muscimol + baclofen microinjections (VP inactivation group; $n=16$, eight male, eight female), or vehicle microinjections (control group) or nothing ( $n=10$, four male, six female) on the third day. All rats were placed in taste reactivity chambers immediately following microinjections.

Twenty-five min after receiving microinjections, rats were video-recorded in taste reactivity chambers for $5 \mathrm{~min}$ to assess spontaneous behavior prior to oral sucrose solution infusion ( $n$ $=20$; seven male, thirteen female). Cameras viewed and 


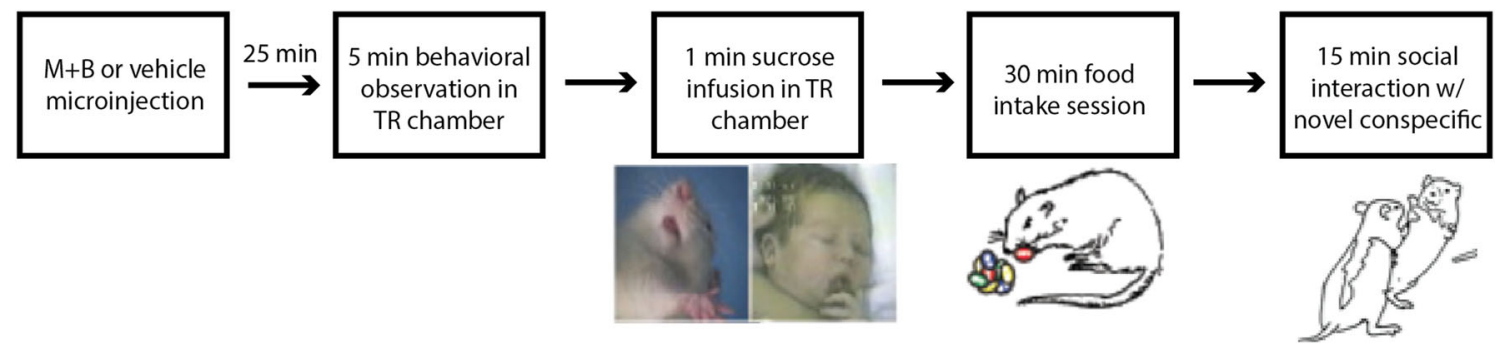

Fig. 1 Experimental schedule. Rats received several behavioral tests after microinjection of either muscimol + baclofen $(\mathrm{M}+\mathrm{B})$ or vehicle in $\mathrm{pVP}$. First, rats were placed in taste reactivity chambers. Twenty-five min after receiving microinjections, rats were video-recorded for $5 \mathrm{~min}$ to assess spontaneous behavior. Thirty min after receiving microinjections, rats underwent taste reactivity testing to an oral sucrose infusion $(1 \mathrm{~mL} / 1$ min) while their orofacial reactions were video-recorded via close-up

recorded rats via an angled mirror placed under the transparent floor of the taste reactivity chamber. Videos were scored offline at normal speed using Observer software (Noldus, Netherlands) for mouth movements, tongue protrusions, gaping, grooming, rearing, cage crossing, and hyperkinetic treading.

\section{Taste reactivity testing and video scoring}

Thirty min after microinjections and immediately after the 5min spontaneous behavior sample, rats were tested in the taste reactivity paradigm to measure affective orofacial reactions to $1 \mathrm{~mL}$ of a $1 \%$ sucrose solution infused into the mouth continuously for $1 \mathrm{~min}$. To deliver solution to the mouth, a $10 \mathrm{~mL}$ syringe containing sucrose solution in a syringe pump was attached to PE-50 tubing with a PE-10 delivery nozzle that could be inserted into a rat's oral cannula. Orofacial taste reactivity responses were recorded in a similar manner as described above, but with close-ups of the rat's face to better assess orofacial reactions.

Taste reactivity videos were scored offline at $1 / 5$ th of actual speed using Observer software. Rats were scored for positive hedonic responses, negative aversive responses, and neutral responses. Hedonic responses were classified as rhythmic midline tongue protrusions, lateral tongue protrusions, and paw licks. Aversive "disgust" responses were classified as gapes, head shakes, face washes, forelimb flails, and chin rubs. Although associated with aversion, hyperkinetic paw treading was not included in "disgust" counts. Neutral responses were classified as passive dripping of solution from the mouth, grooming, rearing, and rhythmic mouth movements (Berridge, 2000). A time-bin scoring system was used to ensure that low-frequency behaviors (e.g., lateral tongue protrusions) contribute equally to the hedonic or aversive score of each animal as do high-frequency behaviors (e.g., rhythmic tongue protrusions). Rhythmic mouth movements, passive dripping, hyperkinetic treading, and paw licking reactions were scored in 5-s time bins. Rhythmic midline tongue camera from a transparent floor below. Then, rats were moved to food intake chambers containing palatable food and cob bedding for a 30-min voluntary intake test and were video-recorded for behavior. Finally, a social interaction test was conducted in which a novel conspecific partner was placed in the chamber, and both rats were video-recorded and assessed for social interactions during a 15-min test. All videos were subsequently scored off-line

protrusions and chin rubs were scored in 2-s time bins. Lateral tongue protrusions, gapes, forelimb flails, and head shakes were scored as discrete events and thus single occurrences, which is equivalent to a time-bin score of $1 \mathrm{~s}$. Hedonic reaction totals consisted of the cumulative score of lateral tongue protrusions, rhythmic tongue protrusions, and paw licks. Rhythmic tongue protrusions and paw licks were scored together as they often occurred concurrently. Aversive "disgust" reaction totals consisted of the cumulative score of gapes, head shakes, forelimb flails, and chin rubs.

\section{Voluntary food intake test}

Immediately after taste reactivity testing, rats were transferred to rectangular Plexiglass chambers $(40 \mathrm{~cm} \times 40 \mathrm{~cm} \times 45.7 \mathrm{~cm})$ containing crushed corn cob bedding on the floor, and dishes containing preweighed palatable milk chocolate candy (Mars M\&Ms, $20 \mathrm{~g}$ ). Behavior was video recorded for $30 \mathrm{~min}$, for later scoring. Chocolate intake was measured by weighing M\&Ms after testing and subtracting from pretest weight. Videotaped behavior was scored off-line for feeding behavior and aversive psychomotor behavior (chin rubbing, head burrowing, hyperkinetic treading, and face washing) using Observer software on $1 \times$ to $4 \times$ actual speed. Feeding behavior was classified as the total time spent sniffing, carrying, and eating palatable chocolates. Hyperkinetic treading involved successive rhythmic full-length forelimb extension on the floor followed by retraction, with left and right forelimbs in alternation, as reported in previous studies of VP lesions (Berridge \& Cromwell, 1990). Hyperkinetic treading resembles "disgust"-induced "paw treading," and differs from defensive or fearful treading/burying, which serves to spray bedding toward a stimulus perceived as threatening (Reynolds \& Berridge, 2001). Hyperkinetic treading keeps the paws firmly planted, pushing downward on the floor and is often accompanied by chin rubs (Berridge \& Cromwell, 1990), whereas defensive treading tilts the paws vertically and oriented in front to shovel bedding forward, and is not accompanied by 
chin rubs (Reynolds \& Berridge, 2001). Head burrowing behavior here also was often accompanied by chin rubs, in which the chin was planted firmly on the floor as the rat pushed its head and front body forward through and under the cob bedding; it was similarly considered to be a potentially aversive reaction.

\section{Social interaction test}

After the 30-min food intake test, food was removed from Plexiglass chambers, and a novel same-sex conspecific was added to the chamber to assess social interaction behaviors between the two rats. Behavior was video recorded for $15 \mathrm{~min}$ and scored using Observer software at normal speed, and subsequently scored off-line for prosocial, indifferent/antisocial, and aversive psychomotor behaviors. Prosocial behavior was classified as the total time test rats spent approaching and in physical contact while nuzzling, mounting, pinning, and sniffing the body and genitals of the other rat. Indifferent/antisocial behavior was classified as the total time test rats spent passively receiving approaches from the other rat without responding in return, or in actively turning away or escaping from the other rat's approach. Aversive psychomotor behavior was classified as the total time test rats spent chin rubbing, head burrowing, hyperkinetic treading, and face washing.

\section{Hunger-satiety comparison}

A separate group of rats ( $n=10$; four male, six female) was implanted with the same intracranial VP microinjection guide cannulae and with oral cannulae, and was later tested both in a satiety (ad libitum access to food condition) and in a hungry condition (in which rats had 24-hr restricted access to chow pellets; water was always available ad libitum). This was done in order to assess whether hunger reduces or modulates the degree of sucrose "disgust" induced by VP inactivation after muscimol + baclofen microinjections, given that an early brief report suggested that hunger might alleviate excessive disgust during recovery from large lateral hypothalamic lesions (Fluharty \& Grill, 1981). All rats underwent sucrose taste reactivity tests in four counterbalanced states/VP conditions: vehicle microinjection in ad libitum condition, vehicle microinjection after food restriction, muscimol + baclofen microinjection in ad libitum condition, and muscimol + baclofen microinjection after food restriction. Taste reactivity testing and scoring followed the same procedures described above.

\section{Histology and immunohistochemistry}

Rats were euthanized 120 min after their final muscimol + baclofen or vehicle microinjection and taste reactivity test with sucrose, via sodium pentobarbital overdose, and were transcardially perfused with isotonic saline followed by sodium phosphate-buffered $4 \%$ formaldehyde. Brains were removed and post-fixed in the formaldehyde solution for 3 days, and then transferred to a $25 \%$ sucrose solution for 2-4 days prior to sectioning. Brains were cryostat sectioned, and slices were collected in three series of $50 \mu \mathrm{m}$ coronal sections. Sections were stored in cryoprotectant and placed in a $-20^{\circ} \mathrm{C}$ freezer until retrieved for staining.

Immunofluorescence was used to visualize Fos protein. All antibodies used for immunohistochemistry were diluted in $0.1 \mathrm{M}$ sodium phosphate buffer (NaPB, $\mathrm{pH} 7.4$ ) with $2.5 \%$ donkey serum, $0.2 \%$ Triton $\mathrm{X}-100$, and $0.05 \%$ sodium azide. All rinse steps were performed with $\mathrm{NaPB}$ at 5-min intervals over the course of $15 \mathrm{~min}$. All steps were performed at room temperature. Sections were rinsed to remove cryoprotectant, incubated in goat anti-c-fos (1:1000, Santa Cruz Biotechnology, cat. \#SC-52) overnight, rinsed, incubated in biotinylated donkey antigoat $(1: 300$, Jackson ImmunoResearch, cat. \#705-065-147) for 2 hours, rinsed, incubated in streptavidin-Cy3 (1:300 Jackson ImmunoResearch, cat. \#016-160-084) for 1.5 hours, rinsed in distilled water, incubated in DAPI dihydrochloride $(0.5 \mathrm{ug} / \mathrm{mL}$ in distilled water, Sigma Aldrich, cat. \#D9542) for $20 \mathrm{~min}$ to counterstain, rinsed, mounted onto slides with polyvinyl alcohol mounting medium, briefly dried, and coverslipped.

\section{Imaging, Fos quantification, and Fos antiplume mapping}

Digital images of coronal brain sections were taken using a CCD camera (FX1520, SPOT Imaging Solutions, Sterling Heights, MI, USA) attached to a Leica microscope (DM400B, Leica, Wetzlar, Germany) with fixed camera settings at $5 \times$ magnification. Brain sections were scanned for Fos immunofluorescence and whole-section images were taken in grayscale to provide clearer contrast between Fos expression and basal expression. Fos immunoreactive cells were identified as fluorescent oval-shaped nuclei and counted using ImageJ software (National Institutes of Health). Brain regions were delineated for analysis by comparing images with two prominent rat brain atlases and identifying region-specific landmarks (Paxinos \& Watson, 2007; Swanson, 2004). The specific brain structures and their anteroposterior extents sampled relative to bregma are shown in Table 1: Cortex (medial orbitofrontal cortex, lateral orbitofrontal cortex, ventral orbitofrontal cortex, anterior cingulate cortex, infralimbic/prelimbic cortex, anterior insula, posterior insula); Amygdala (central nucleus, basolateral nucleus); Nucleus accumbens (anterior core, posterior core, anterior medial shell, posterior medial shell); Neostriatum (anterodorsal, anteroventral, posterodorsal, and posteroventral quadrants); Thalamus (anterior paraventricular nucleus, posterior paraventricular nucleus); Lateral habenula; Subthalamic nucleus; Basal nucleus of the stria terminalis; Anterior ventral pallidum; Hypothalamus (paraventricular nucleus, perifornical 
Table 1 Brain regions sampled for Fos immunoreactivity

\begin{tabular}{|c|c|c|}
\hline Brain Region & Range Sampled (mm AP relative to bregma) & Dimensions of Brain Region Sampled (um x um) \\
\hline ACC & +2.52 to +4.20 & $1088 \times 640$ \\
\hline $\mathrm{mOFC}$ & +4.20 to +4.68 & $768 \times 1024$ \\
\hline vOFC & +4.20 to +4.68 & $1088 \times 1280$ \\
\hline 1OFC & +4.20 to +4.68 & $1024 \times 1024$ \\
\hline IL/PrL & +3.00 to +4.20 & $1152 \times 2304$ \\
\hline aInsula & +2.04 to +2.52 & $1152 \times 1152$ \\
\hline aNAcSh & +2.28 & $384 \times 576$ \\
\hline pNAcSh & +1.08 & $512 \times 1024$ \\
\hline aNAcC & +2.28 & $576 \times 1024$ \\
\hline pNAcC & +1.08 & $448 \times 640$ \\
\hline $\mathrm{aDS}$ & +1.80 to +2.28 & $1792 \times 1024$ \\
\hline $\mathrm{aVS}$ & +1.80 to +2.28 & $896 \times 1152$ \\
\hline $\mathrm{pDS}$ & -1.32 to -1.80 & $1024 \times 2048$ \\
\hline pVS & -1.32 to -1.80 & $896 \times 2176$ \\
\hline $\mathrm{aVP}$ & +0.72 to +0.12 & $832 \times 640$ \\
\hline BNST & +0.60 to -0.12 & $320 \times 448$ \\
\hline $\mathrm{PVH}$ & -1.44 to -2.04 & $576 \times 384$ \\
\hline pInsula & -1.08 to -2.28 & $1536 \times 1536$ \\
\hline VMH & -2.04 to -3.00 & $448 \times 448$ \\
\hline $\mathrm{CeA}$ & -2.04 to -2.64 & $448 \times 448$ \\
\hline $\mathrm{LHb}$ & -3.00 to -3.24 & $512 \times 384$ \\
\hline PeFLH & -3.00 to -3.48 & $896 \times 896$ \\
\hline PLH & -3.00 to -3.48 & $576 \times 704$ \\
\hline $\mathrm{TuLH}$ & -3.00 to -3.48 & $320 \times 320$ \\
\hline DMH & -3.24 & $640 \times 896$ \\
\hline aPVT & -1.32 & $640 \times 1024$ \\
\hline pPVT & -3.24 & $640 \times 640$ \\
\hline STh & -3.60 to -3.96 & $1664 \times 1280$ \\
\hline SuM & -4.36 to -4.92 & $2880 \times 768$ \\
\hline pSNR & -6.24 & $1920 \times 1920$ \\
\hline VTA & -6.24 & $1024 \times 1024$ \\
\hline $\mathrm{PBN}$ & -9.00 to -9.84 & $1152 \times 768$ \\
\hline NTS & -12.24 to -14.04 & $640 \times 384$ \\
\hline
\end{tabular}

Note. Fos-immunoreactive neurons were counted in all brain regions listed to identify neural activation changes recruited by muscimol + baclofen microinjections into the pVP that induced excessive "disgust" reactions to oral sucrose, and then compared to corresponding counts measured in other brains after vehicle microinjections as a control baseline. The "range sampled" column shows the extent in stereotaxic atlas coordinates as mm anteroposterior relative to bregma. The "dimensions" column shows the box size of coronal section tissue in which Fos-expressing neurons were counted for that structure. Structure abbreviations: $\mathrm{ACC}=$ anterior cingulate cortex; $\mathrm{mOFC}=$ medial orbitofrontal cortex; vOFC = ventral orbitofrontal cortex; $1 \mathrm{OFC}=$ lateral orbitofrontal cortex; IL/PrL = infralimbic/prelimbic cortex; aInsula = anterior insula cortex; pInsula = posterior insula cortex; aNAcSh = anterior nucleus accumbens shell; $\mathrm{pNAcSh}=$ posterior nucleus accumbens shell; $\mathrm{aDS}=$ anterodorsal neostriatum; $\mathrm{aVS}=$ anteroventral neostriatum; $\mathrm{pDS}=$ posterodorsal neostriatum; $\mathrm{pVS}=$ posteroventral neostriatum; $\mathrm{aVP}=$ anterior ventral pallidum; $\mathrm{BNST}=$ bed nucleus of stria terminalis; CeA = central amygdala; PVH = paraventricular nucleus of hypothalamus; PeFLH = perifornical nucleus of hypothalamus; PLH = peduncular nucleus of lateral hypothalamus; TuLH = tuberal nucleus of lateral hypothalamus; $\mathrm{DMH}=$ dorsomedial nucleus of hypothalamus; $\mathrm{LHb}=$ lateral habenula; $\mathrm{aPVT}=$ anterior paraventricular nucleus of thalamus; $\mathrm{pPVT}=$ posterior paraventricular nucleus of thalamus; $\mathrm{STh}=$ subthalamic nucleus; $\mathrm{SuM}=$ supamammillary nucleus; pSNR = posterior substantia nigra; VTA = ventral tegmental area; $\mathrm{PBN}=$ parabrachial nucleus of pons; $\mathrm{NTS}=$ nucleus of solitary tract

nucleus, supramammillary nucleus, peduncular nucleus of the lateral hypothalamus); Midbrain (ventral tegmental area, posterior substantia nigra); Hindbrain (nucleus of the solitary tract of the medulla, parabrachial nucleus of the pons).
Local Fos plumes in pVP Drug microinjections produce local plumes of altered Fos expression immediately surrounding the microinjector tip, which reflect direct alterations in neuronal function caused by the microinjected drug. The plume size 
thus reveals the location and spread of drug impact on local neurons. Drugs that stimulate Fos expression create excitatory "Fos plumes," and drugs that suppress neuronal translation of Fos produce what have been called inhibitory "Fos antiplumes." As muscimol + baclofen should inhibit neuronal activity, we expected to see local antiplumes within the pVP after muscimol + baclofen microinjection when compared with vehicle injection levels as control baseline (Ho \& Berridge, 2014). The average shape and size of Fos antiplumes was assessed in a group of rats that was randomly selected from our experiments $(n=8)$. Fos plumes were visualized at $5 \times$ to $10 \times$ magnification under a microscope and captured by digital camera. In image editing software (GIMP), the injection site image was overlaid with $50 \mu \mathrm{m} \times$
$50 \mu \mathrm{m}$ sampling blocks placed along seven radial arms emanating from the center of the microinjection site $\left(45^{\circ}, 90^{\circ}\right.$, $135^{\circ}, 180^{\circ}, 225^{\circ}, 270^{\circ}$, and $315^{\circ}$ ) at $50 \mu \mathrm{m}$ intervals, adding to 15 blocks per radial arm (Richard \& Berridge, 2011). The number of neurons expressing Fos within each box were counted manually by a counter blind to experimental condition (see Fig. 2c-d for examples) and added to a spreadsheet for calculations. The average maximal radii containing $<75 \%$ and $<50 \%$ Fos expression of vehicle levels (i.e., $25 \%$ suppression and 50\% suppression below control baselines measured in the corresponding box after a vehicle microinjection) were calculated. Those inner and outer plume radii were used to determine the outer and inner sizes of symbols in stereotaxic maps of pVP sites (see Fig. 2a; see Fig. 3 for examples). a

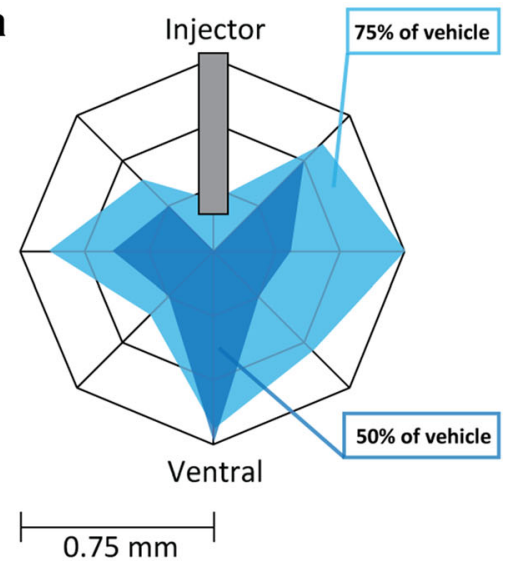

Average maximal outer anti-plume radius: $0.53 \mathrm{~mm}$ Average maximal inner anti-plume radius: $0.34 \mathrm{~mm}$

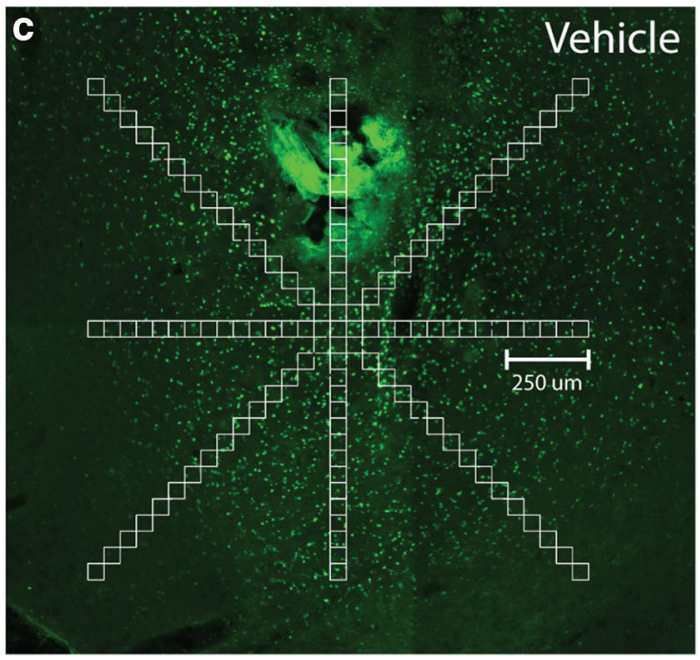

Fig. 2 Local Fos antiplumes of Fos suppression produced in pVP by muscimol + baclofen microinjection. a The antiplume shape was calculated by contrasting average Fos counts for muscimol + baclofen versus vehicle groups. Example map of suppression zones (light blue = $<75 \%$ of vehicle control level (but $>50 \%$ control levels); dark blue $=$ $<50 \%$ control level). b Quantitative degree of local Fos suppression in $\mathrm{pVP}$ as a function of distance from microinjection center (100\% dashed

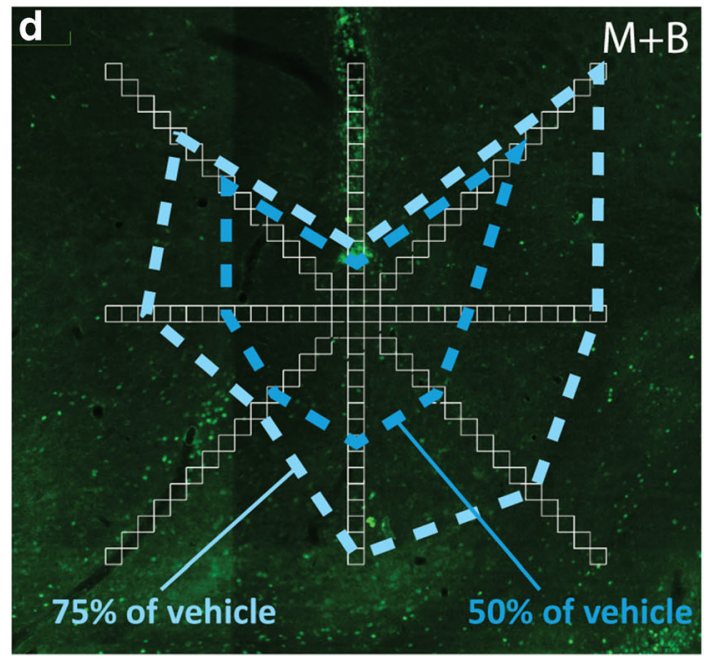

line $=$ vehicle control levels at that distance point). Suppressive effects of drug microinjection decrease with distance away from center point. c Radial arm overlays were superimposed on pVP microinjection site, and the number of local Fos-expressing neurons was counted in each box. $\mathrm{d}$ Example of local suppression in $\mathrm{pVP}$ surrounding microinjection site of muscimol + baclofen. Dashed lines show boundaries of suppression zones in this rat, compared with average vehicle control levels 


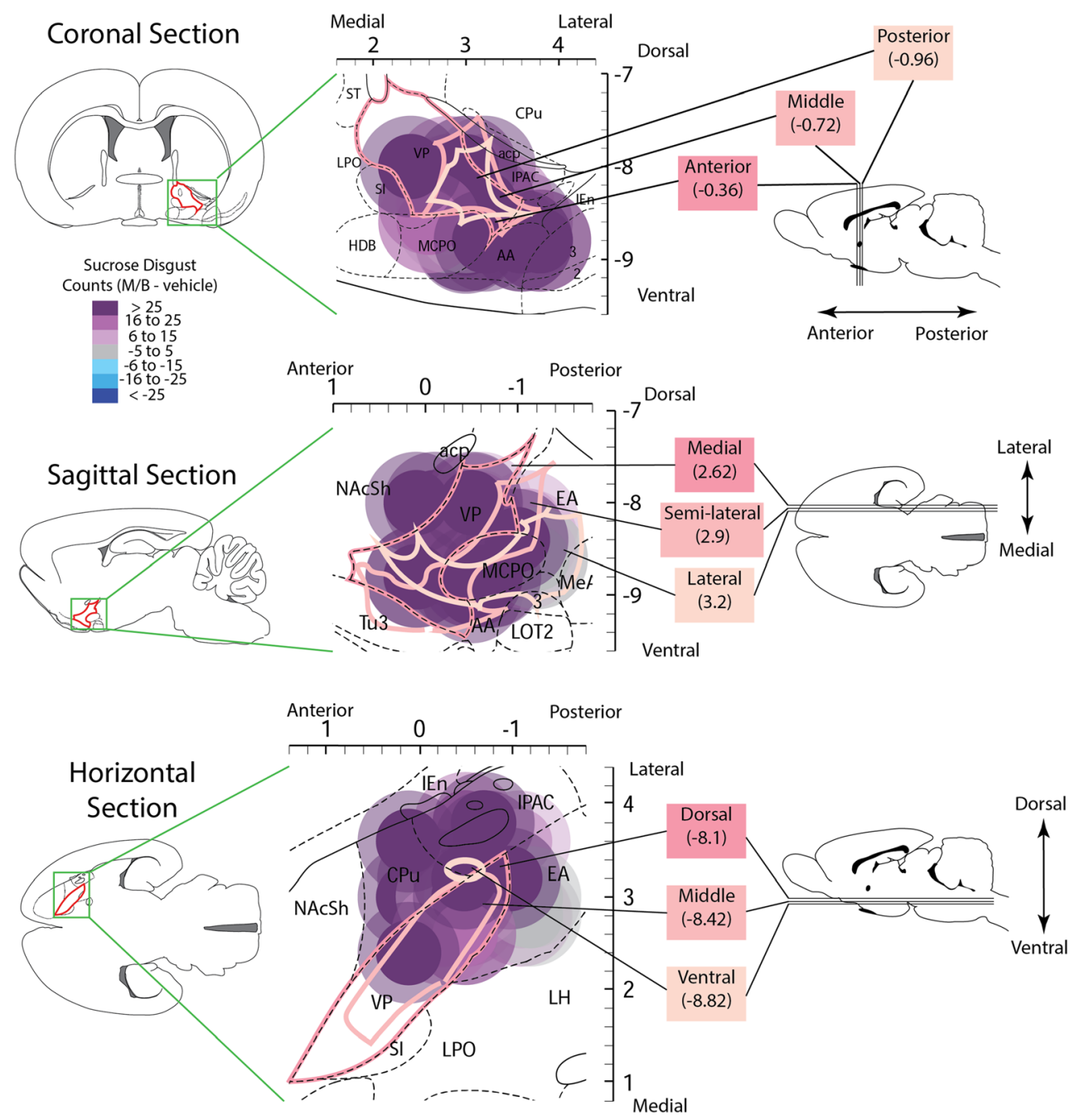

Fig. 3 Plume maps of "disgust" induction sites in and around pVP. Colors of plume symbols show the magnitude of "disgust" reactions to sucrose taste after muscimol + baclofen microinjection at that site: purple denotes strong "disgust", or $>600 \%$ compared with after vehicle in the same rats; mean $(\mathrm{M}+\mathrm{B})=27.9$ versus mean $($ vehicle $)=4.06 ; t(15)=6.89$, $p<.001$, Cohen's $d=2.56$. Top shows a coronal plane $(0.36$ to $0.96 \mathrm{~mm}$ posterior to bregma), middle shows a sagittal plane (2.62 to $3.2 \mathrm{~mm}$ lateral to bregma), and bottom shows a horizontal plane (8.1 to $8.82 \mathrm{~mm}$ below the skull surface) view of the same pVP sites. $\mathrm{AA}=$

\section{Statistical analysis}

Statistical testing was performed using SPSS software (IBM). A mixed two-factor ANOVA was used to detect significant effects of treatment, sex, or their interaction on behavior. Eta squared $\left(\eta^{2}\right)$, calculated as sum of squares of the factor of interest divided by the total sum of squares, was used as a measure of effect size of these ANOVA factors. All behavioral and histological paired comparisons were two-tailed Student's $t$ tests with unequal variances assumed, and alpha was set at $p<.05$ for all analyses. Independent-samples $t$ tests were used to compare behavioral and histological data between experimental and vehicle-only groups, and paired $t$ tests were used to compare behavioral data within groups. Levene's test for equality was used to determine anterior amygdala; NAcSh $=$ nucleus accumbens shell $;$ acp $=$ posterior part of anterior commissure; $\mathrm{CPu}=$ caudate putamen; $\mathrm{HDB}=$ horizontal limb of the diagonal band; IEn = intermediate endopiriform nucleus; IPAC $=$ interstitial nucleus of the posterior part of the anterior commissure; LOT2 = lateral olfactory nucleus layer $2 ; \mathrm{LPO}=$ lateral preoptic area $\mathrm{MeA}=$ medial amygdala $\mathrm{MCPO}=$ magnocellular preoptic area; MPO = medial preoptic area; $\mathrm{SI}=$ substantia innominata; $\mathrm{ST}=$ bed nucleus of stria terminalis; Tu3 = olfactory tubercle layer 3

whether equal or unequal variances could be assumed for independent-samples $t$ tests; presence of Levene's $p$ value (alpha $<0.05$ ) below indicates unequal variance and that Welch's $t$ test was used. Cohen's $d$ statistic was computed to measure effect size for all comparisons.

\section{Results}

\section{Fos antiplume characterization and VP localization of behavioral effects}

Microinjections of muscimol + baclofen in $\mathrm{pVP}$ produced small local Fos antiplumes of suppressed Fos expression in 
pVP, immediately surrounding each microinjection site. Fos suppression was most intense at the center of the microinjection site, suppressing expression within a radius of $0.34 \mathrm{~mm}$ by more than $50 \%$ below vehicle-control baselines that were measured at corresponding sites in control rats that received pVP microinjections of only vehicle without muscimol + baclofen. This Fos suppression strength was inversely related to increasing distance away from the center, as local drug impact diminished with diffusion (see Fig. 2a-b). Thus, inhibitory Fos antiplumes in pVP had an inner radius of $0.34 \mathrm{~mm}$ of $>50 \%$ suppression of Fos expression (i.e., less than half vehicle-baseline level of Fos), and a mean outer radius of $0.53 \mathrm{~mm}$ of $>25 \%$ suppression (i.e., $50 \%-75 \%$ of baseline Fos). Photomicrograph comparisons of vehicle and Fos expression at pVP injection sites can be seen in Fig. 2c-d.

\section{Excessive "disgust" from pVP "hot spot" inactivation} Temporary inactivation of pVP neurons by muscimol + baclofen microinjections eliminated normal "liking" reactions to sucrose infusions into the mouth, instead replacing them with excessive "disgust reactions" such as gapes, chin rubs and paw treading, which were repeated nearly continuously in rapid and vigorous succession as long as the sucrose infusion continued. "Disgust" reactions were $>600 \%$ higher after muscimol + baclofen microinjections than near-zero levels elicited by sucrose from the same rats after control vehicle microinjections, $t(15)=6.893, p<.001$, Cohen's $d=2.56$ (see Fig. 4). Gapes were elicited at $>1,900 \%$ vehicle control levels, $t(15)=7.143, p<.001, d=2.40$, and chin rubs at $>600 \%$ control levels, $t(15)=7.083, p<.001, d=2.50$. By contrast, after vehicle microinjections, sucrose tastes elicited only positive hedonic orofacial reactions from all rats, such as lateral tongue protrusions and paw licks, Exp group with vehicle vs. Ctrl group with vehicle: $t(24)=1.248, p=.224, d=$ 0.511 . Those positive "liking" reactions were completely abolished after muscimol + baclofen microinjections, Exp group with muscimol + baclofen vs. Ctrl group with vehicle: $t(9)=-8.916, p<.001, d=-3.98$; Exp group with muscimol + baclofen vs. Exp group with vehicle: $t(15)=-9.089, p<$ $.001, d=-3.25$.

Female and male rats were similarly positive to sucrose after vehicle, and similarly negative after muscimol + baclofen, so there were no significant sex differences, Hedonic: $F(1,14)=0.195, p=.666, \eta^{2}=0.002$; Aversive, $F(1,14)=$ $1.219, p=.288, \eta^{2}=0.016$, nor did sex significantly interact with microinjection treatment, Hedonic: $F(1,14)=0.195, p=$ $.666, \eta^{2}=0.002$; Aversive: $F(1,14)=2.389, p=.144, \eta^{2}=$ 0.027 , and sex alone did not have a significant effect on either of these measures.

Potentiation of paw treading within constellation of "disgust" reactions Microinjections of muscimol + baclofen into pVP also reconfigured the frequency hierarchy of "disgust" reactions in a sensorimotor fashion, by specifically promoting the

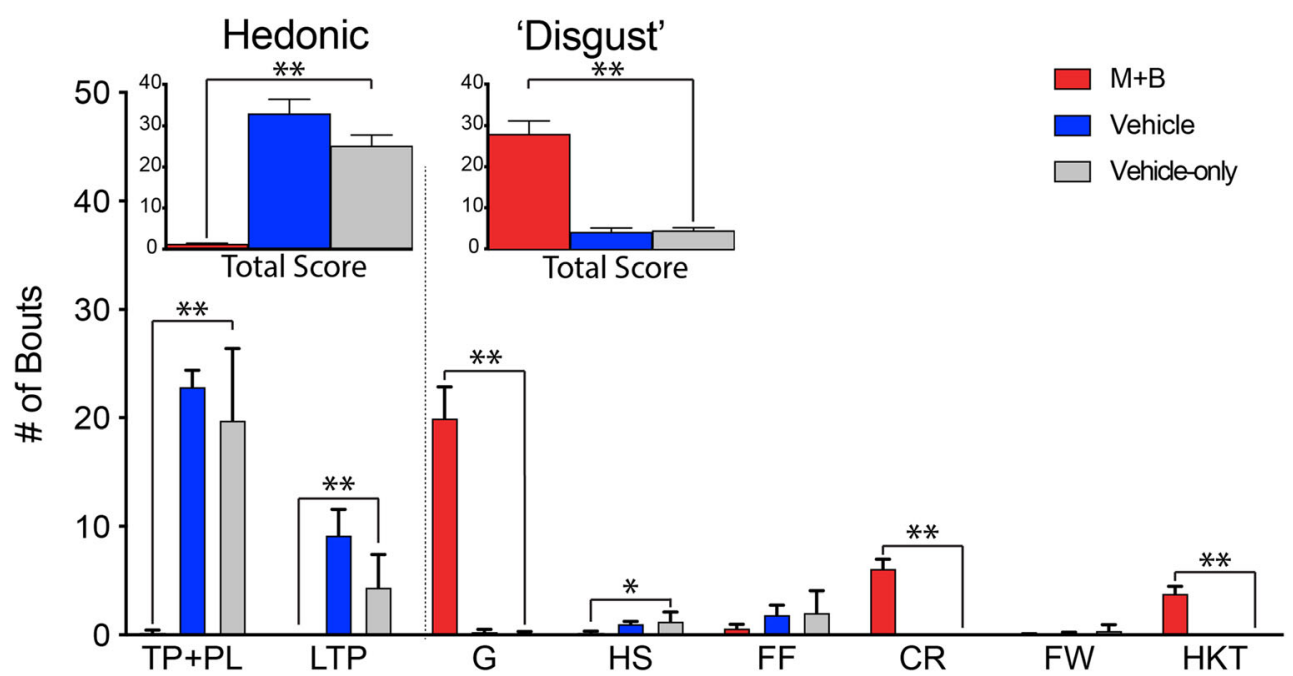

Fig. 4 Sucrose taste elicited positive "liking" reactions after vehicle control microinjection, but instead elicited only excessive "disgust" reactions after $\mathrm{pVP}$ inactivation via muscimol + baclofen $(\mathrm{M}+\mathrm{B})$ microinjection in the same rats. Positive hedonic orofacial reactions ("liking") elicited after vehicle (blue) or muscimol + baclofen (red) microinjections in $\mathrm{pVP}$. Also shown are sucrose reactions of the Fos control group that received only vehicle microinjections (gray). Following muscimol + baclofen microinjection, sucrose taste increased "disgust" reactions by $>600 \%, t(15)=6.893, p<.001, d=2.56$, and abolished hedonic orofacial reactivity, $t(15)=-9.089, p<.001, d=-3.25$, compared with vehicle microinjection in the same rats. Separate positive hedonic component reactions are rhythmic midline tongue protrusions
(TP), paw licks (PL), and lateral tongue protrusions (LTP). Tongue protrusions and paw licks were scored together as they often occurred concurrently. "Disgust" reaction components are gapes $(\mathrm{G})$, head shakes (HS), forelimb flails (FF), chin rubs (CR), and face washing (FW). Hyperkinetic paw treading (HKT), although associated with "disgust," was not included in these total "disgust" counts to avoid inflation due to sensorimotor potentiation. Following muscimol + baclofen microinjection, sucrose taste increased gapes by $>1,900 \%, t(15)=7.143, p<.001, d$ $=2.40$, and chin rubs by $>600 \%, t(15)=7.083, p<.001, d=2.50$, compared with the near-zero levels observed upon vehicle microinjection in the same rats. $* p<.05 . * * p<.005$. (Color figure online) 
incidence, number and form of forepaw treading. In normal rats, paw treading is a "disgust" reaction that is relatively rare compared with gapes, headshakes, chin rubs, and forelimb flails. But excitotoxin lesions of pVP and nearby globus pallidus regions have been reported to alter that response hierarchy, specifically potentiating paw treading into the most frequent, numerous, and persistent single "disgust" reaction, and simultaneously exaggerate the motor trajectories of the paws backward and forward in left-right treading movements, making the treading appear hyperkinetic in its motor pattern (Berridge \& Cromwell, 1990; Cromwell \& Berridge, 1994). Here, muscimol + baclofen inactivations of pVP produced similar hyperkinetic forepaw treading and sensorimotor restructuring, and hyperkinetic treading typically occurred in concert with other aversive behaviors like chin rubbing and head burrowing. Further, hyperkinetic treading in the $5 \mathrm{~min}$ prior to sucrose infusion appeared to grow over time for rats that received two trials of sucrose taste reactivity after muscimol + baclofen microinjections in pVP. Specifically, the duration of hyperkinetic paw treading increased $>275 \%$ between the first and second trials in the $5 \mathrm{~min}$ prior to sucrose infusion, from a mean of $71.2 \mathrm{~s}$ to a mean of $199.2 \mathrm{~s}, t(5)=$ $3.660, p=.014, d=1.32$. This increase in hyperkinetic treading appeared to require both muscimol + baclofen microinjection and experience with sucrose as "disgusting," as it did not occur in rats who similarly underwent two trials with muscimol + baclofen in pVP but lacked oral cannulae and never experienced sucrose infusion under its influence, $t(5)=-0.671, p=$ $.532, d=-0.14$. Similarly, even in rats used for sucrose testing, the anticipatory occurrence of treading before the second sucrose infusion began did not occur in rats who were placed in a novel taste reactivity chamber (different from the one used in their first taste reactivity test) for their second trial of sucrose after muscimol + baclofen microinjection, $t(2)=0.236, p=$ $.835, d=-0.19$, suggesting it could occur as a Pavlovian conditioned reaction to association with sucrose "disgust," as well as an unconditioned response to actual "disgusting" sucrose.

Posterior VP hot spot is the anatomical release site for sweetness "disgust." Fos antiplumes and sites of muscimol + baclofen microinjections were examined to find any underlying common anatomical substrate shared by those that evoked excessive "disgust" reactions (see Fig. 3). The plume centers of most disgust-inducing sites were within $\mathrm{pVP}$, though a few sites had plume centers outside VP, yet nearby. Examination of these outlying sites indicated that their Fos antiplumes still at least partly encroached upon the pVP, particularly the 0.53$\mathrm{mm}$ radius of the outer Fos antiplume of $>25 \%$ suppression, and at least for one of the two bilateral microinjections in that rat. Therefore, overall, our data appear consistent with previous reports that excessive "disgust" reactions are released primarily by impairing neurons within the pVP subregion (Cromwell \& Berridge, 1993; Ho \& Berridge, 2014).
Several other microinjection sites, which had centers positioned slightly more distant from $\mathrm{pVP}$ and nearer to the lateral hypothalamus or amygdala such that their Fos plumes did not encroach on $\mathrm{pVP}$, failed to induce intense "disgust" to sucrose. However, all sites at least completely suppressed positive hedonic or "liking" reactions to sucrose taste after muscimol + baclofen microinjections (see Fig. 4). Whether reduced "liking" resulted from less intense inactivation of $\mathrm{pVP}$ neurons (i.e., $<25 \%$ suppression of Fos levels), or instead from stronger inactivation of hypothalamic or extended amygdala neurons, cannot be confidently decided based on our data.

VP "disgust" remained stable in hunger state All rats above had been tested in a food-sated state, always having free access to unlimited food as well as water in their home cage. To test whether excessive sucrose "disgust" persisted when rats were tested in a physiologically hungry state, a subgroup of rats was given a 24-hr period of food restriction prior to $\mathrm{pVP}$ microinjections of muscimol + baclofen and a taste reactivity test with sucrose. Results showed that robust "disgust" reactions to sucrose were still induced by $\mathrm{pVP}$ inactivation in the hungry state, and did not significantly differ from "disgust" levels in the same rats when they were tested after pVP inactivation in a nondeprived, food ad libitum state. This suggests that excessive "disgust" induced by pVP inactivation was not detectably mitigated by hunger at this level, Hedonic: $t(9)=$ $-1.115, p=.294, d=-0.53$; Aversive: $t(9)=1.505, p=.167, d$ $=0.61$.

\section{Suppression of motivation to eat: Voluntary food intake test} In voluntary food intake tests conducted immediately after taste reactivity tests, $\mathrm{pVP}$ inactivations by muscimol + baclofen microinjections also completely abolished average consumption of $5.14 \mathrm{~g}$ of chocolate candy that normally followed control vehicle microinjections, reducing consumption to zero, $t(12)=-6.690, p<.001, d=-2.62$. Further, muscimol + baclofen microinjection increased the rats' spontaneous display of several "disgust" taste reactivity components, perhaps elicited by the sight and smell of food in the chamber, or as a persisting after-reaction to the oral sucrose infusion that had been experienced several minutes to 30 minutes earlier. These most prominently included aversive treading, chin rubbing, and head burrowing, all elevated by $>250$-fold compared with levels seen after vehicle microinjection in the same rats, $t(12)$ $=7.265, p<.001, d=2.84$. These behaviors in the presence of food after muscimol + baclofen microinjections typically began with chin rubbing or head burrowing, followed within seconds by bursts of robust hyperkinetic treading as the animal circled the Plexiglass chamber, punctuated by brief bouts of inactivity in which the rat abruptly froze for several seconds.

Female and male sexes again did not differ in intake after either vehicle, $F(1,11)=0.41, p=.842, \eta^{2}<0.001$, or muscimol + baclofen microinjections, $F(1,11)=0.724, p=.413, \eta^{2}=$ 
0.011 , and there was no significant interaction between sex and microinjection treatment, Intake: $F(1,11)=0.41, p=.844, \eta^{2}<$ 0.001; Treading: $F(1,11)=0.706, p=.419, \eta^{2}=0.010$.

\section{pVP inhibition disrupts appetitive social interactions with a conspecific partner}

Rats that received vehicle microinjections into the $\mathrm{pVP}$ readily engaged in social interactions with a novel conspecific partner, especially in the first few minutes of their encounter. The two rats repeatedly directed sniffs to each other's body and anogenital region, and followed each other around the cage. No aggression or avoidance was observed after vehicle microinjections in pVP. Microinjections of muscimol + baclofen in the $\mathrm{pVP}$ caused a $>25$-fold suppression in that rat's prosocial approaches, sniffs, and physical contacts with the other social partner, compared with control levels of behavior of the same rats after vehicle microinjection, $t(10)=-6.265, p<.001, d=$ -2.67 . Muscimol + baclofen in the $\mathrm{pVP}$ also caused a $>2$-fold increase in passively indifferent reactions when the other rat approached, in which the microinjected rat simply remained nearly immobile during an approach without apparently responding in return, $t(10)=5.335, p<.001, d=2.10$. Further, muscimol + baclofen in the pVP also similarly caused a $>4$-fold increase in active negative avoidance or antisocial behavior in which the microinjected rat actively turned away and sometimes took several rapid steps away to escape from the other rat's approach, $t(10)=2.680, p=.02, d=0.94$. Finally, muscimol + baclofen in the pVP also sometimes caused rats to engage in aversive chin rubbing, head burrowing, and hyperkinetic treading during the social situation, just as in the food intake test, at a level $>50$-fold above control vehicle trials, conceivably representing spontaneous "disgust," or else a long-persisting after-reaction to the "disgusting" sucrose infusion 30-45 min earlier, $t(10)=5.041, p=$ $.001, d=2.16$. Males and females responded equivalently in these interactions with a same-sex partner: sexes did not differ overall in social behaviors, Social: $F(1,9)=0.005, p=.944$, $\eta^{2}<0.001$; Antisocial: $F(1,9)=0.233, p=.641, \eta^{2}=0.006$; Aversive: $F(1,9)=0.001, p=.977, \eta^{2}<0.001$, nor did sex significantly interact with $\mathrm{pVP}$ treatment (drug vs. vehicle) to influence any of these patterns, Social, $F(1,9)=0.003, p=$ $.955, \eta^{2}<0.001$; Antisocial: $F(1,9)=0.799, p=.395, \eta^{2}=$ 0.015; Aversive: $F(1,9)=0.003, p=.958, \eta^{2}<0.001$.

\section{pVP inhibition recruits robust Fos expression changes in several limbic regions}

Brains were processed for immunohistology after rats received either $\mathrm{pVP}$ muscimol + baclofen or vehicle microinjection and completed their final experimental trial. Brain anatomical patterns of Fos expression were quantitatively compared via microscope analysis across vehicle versus muscimol
+ baclofen conditions. Results showed that $\mathrm{pVP}$ inactivation, which induced "disgust," recruited robust changes in Fos expression in several limbic and motor-related brain structures, compared with baseline Fos levels demonstrated by the vehicle microinjection control group (see Fig. 5). Overall, increases were found in several subcortical structures, and decreases were found in several cortical structures (see Fig. 6). Subcortical elevations in activation were found in regions of the nucleus accumbens, where induction of pVP "disgust" caused a large $>3,400 \%$ increase in Fos expression in neurons of the posterior half of the medial shell (pNAcSh, Levene's $p$ $=.001), t(8.246)=8.774, p<.001, d=4.14$, which is another site where temporary muscimol + baclofen inactivation can induce excessive "disgust" (Faure et al., 2010; Ho \& Berridge, 2014; Reynolds \& Berridge, 2002). There was also a more moderate $>280 \%$ Fos increase in the anterior core of the nucleus accumbens (aNAcC), $t(14)=2.518, p=.025, d=1.26$, and a trend toward $>280 \%$ Fos increase in the anterior medial shell of the accumbens (which contains another hedonic hot spot), though this last change did not reach significance criterion (aNAcSh), $t(12)=1.862, p=.087, d=1.05$.

In the extended amygdala, we found a Fos increase of $>550 \%$ in the bed nucleus of the stria terminalis (BNST), $t(12)=4.021, p=.002, d=2.29$. In the hypothalamus, Fos increases of $>2,100 \%$ were found in the supramammillary nucleus (SuM, Levene's $p=.001), t(7.108)=6.323, p<$ $.001, d=3.16,>1,100 \%$ in the perifornical nucleus of the hypothalamus (PeFLH, Levene's $p=.006), t(8.496)=$ $7.252, p<.001, d=3.42,>550 \%$ in the paraventricular nucleus of the hypothalamus (PVH, Levene's $p=.002), t(8.908)$ $=6.066, p<.001, d=2.86,>550 \%$ in the dorsomedial hypothalamus (DMH, Levene's $p=.003), t(8.410)=4.793, p=$ $.001, d=2.26$, and $>280 \%$ in the peduncular nucleus of the lateral hypothalamus (PLH, Levene's $p=.004), t(9.550)=$ 2.389, $p=.039, d=1.12$.

In the midbrain, Fos increases of $>3,400 \%$ were found in the ventral tegmental area (VTA, Levene's $p=0.001), t(8.348)$ $=8.995, p<.001, d=4.24$, and posterior substantia nigra $(\mathrm{pSNR}$, Levene's $p=.001), t(8.138)=6.273, p<.001, d=$ 2.96. In the hindbrain, Fos increases of $>1,100 \%$ were found in the medulla's nucleus of the solitary tract (NTS, Levene's $p$ $=.015), t(8.738)=5.311, p=.001, d=2.60$, and $>2,100 \%$ in the parabrachial nucleus of the pons (PBN, Levene's $p<$ $.001), t(8.201)=5.829, p<.001, d=2.75$.

In the thalamus, Fos increases of $>280 \%$ were found in the anterior paraventricular nucleus (aPVT), $t(16)=4.983, p<$ $.001, d=2.35$, and $>550 \%$ in the posterior paraventricular nucleus (pPVT), $t(16)=9.891, p<.001, d=4.66$. Also related to thalamic circuitry, increases of $>1,100 \%$ were found in the lateral habenula (LHb, Levene's $p=.005), t(8.302)=5.818$, $p<.001, d=2.74$, and $>3,400 \%$ in the subthalamic nucleus (STh, Levene's $p<.001), t(7.053)=6.624, p<.001$, $d=3.31$. 

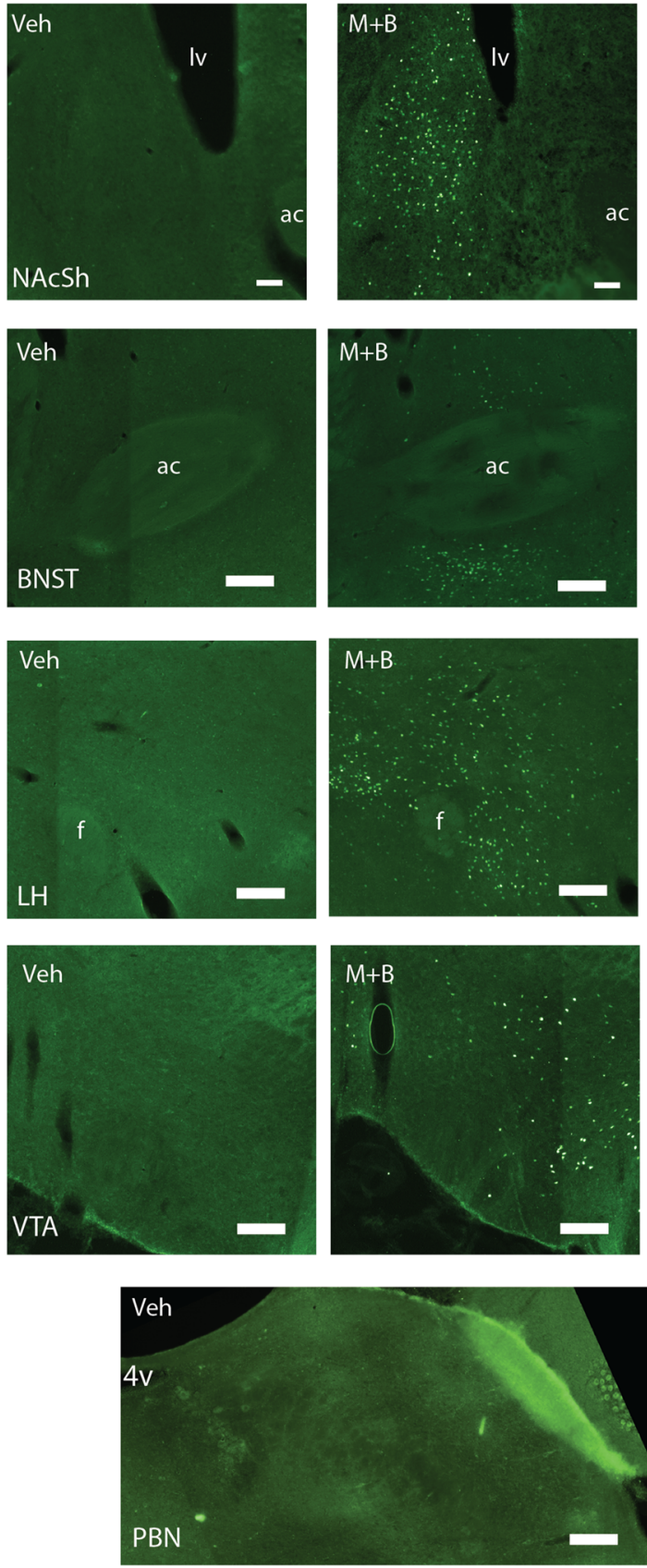

Fig. 5 Photomicrographs of distant Fos expression recruited by excessive "disgust" after inactivation of the pVP. Microscope images show Fos expression in distant brain structures either following $\mathrm{pVP}$ muscimol + baclofen microinjection $(\mathrm{M}+\mathrm{B})$ or following vehicle microinjection (Veh). Structures shown are: posterior shell of the nucleus accumbens (NAcSh), bed nucleus of stria terminalis (BNST), perifornical, peduncular, and tuberal nuclei of lateral hypothalamus (LH), ventral tegmental area (VTA), subthalamic nucleus (STh), substantia nigra (SNR), lateral habenula and posterior paraventricular nucleus of the

Conversely, decreases in Fos expression accompanied pVPinduced "disgust" in several cortical regions related to positive hedonic impact and reward. In orbitofrontal cortex regions, we found a decrease to $<20 \%$ of control levels in the medial orbitofrontal cortex (mOFC, Levene's $p=0.001), t(8.903)=$
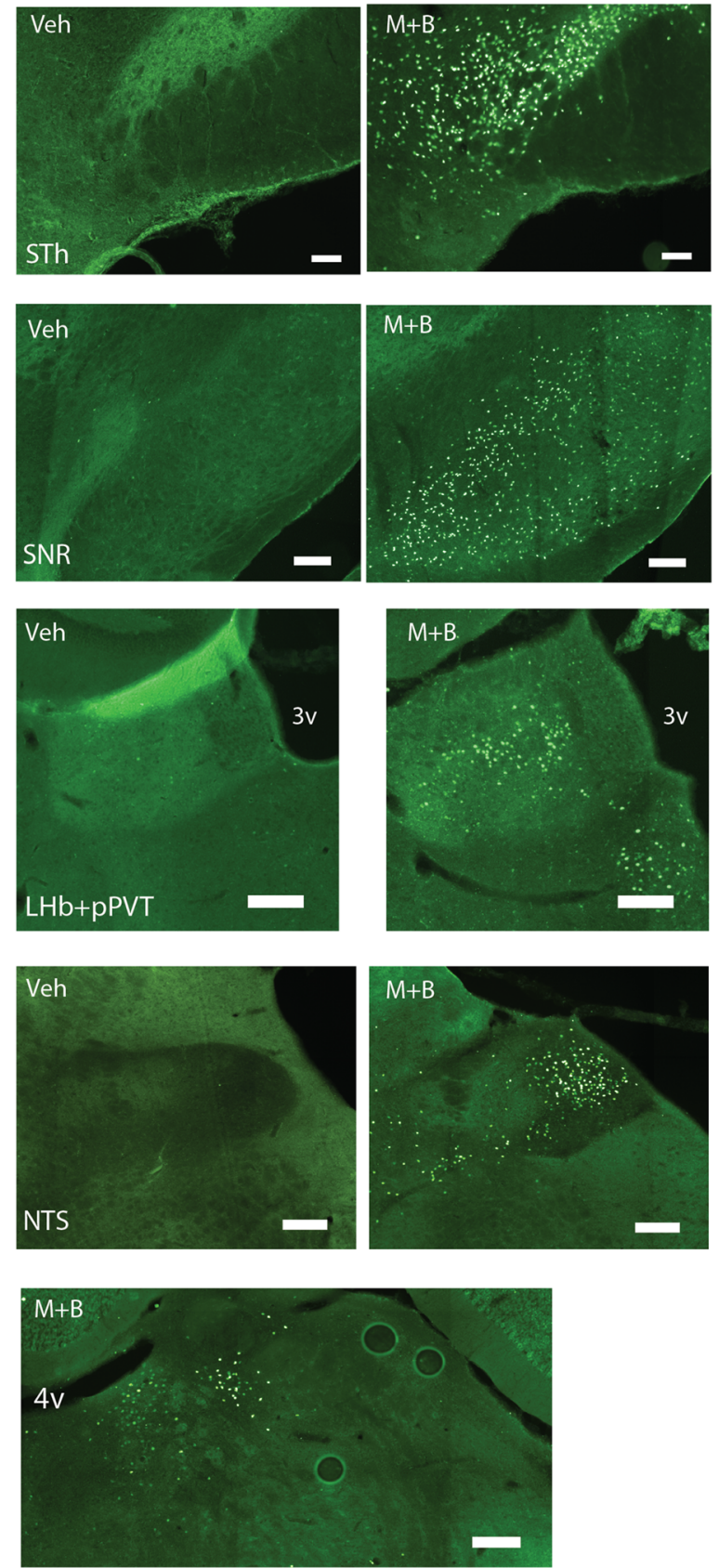

thalamus $(\mathrm{LHb}+\mathrm{pPVT})$, nucleus of the solitary tract (NTS), and parabrachial nucleus of the pons $(\mathrm{PBN})$. All images are unilateral. For the following images, right $=$ lateral and left $=$ medial: NAcSh, LH, VTA, STh, SNR, NTS, PBN. For the following images, right $=$ medial and left $=$ lateral: BNST, LHb+pPVT. Top = dorsal and bottom = ventral for all images. Landmarks shown include the lateral ventricle (lv), anterior commissure (ac), fornix (f), third ventricle (3v), and fourth ventricle (4v). Scale bars represent $200 \mu \mathrm{m}$

$-2.718, p=.024, d=-2.72$, which contains a hedonic hot spot site where mu opioid or orexin microinjection stimulations enhance "liking" reactions to sweetness (Castro \& Berridge, 2017). There was also a $<5 \%$ decrease in the ventral orbitofrontal cortex (vOFC, Levene's $p<.001), t(7.025)=$ 


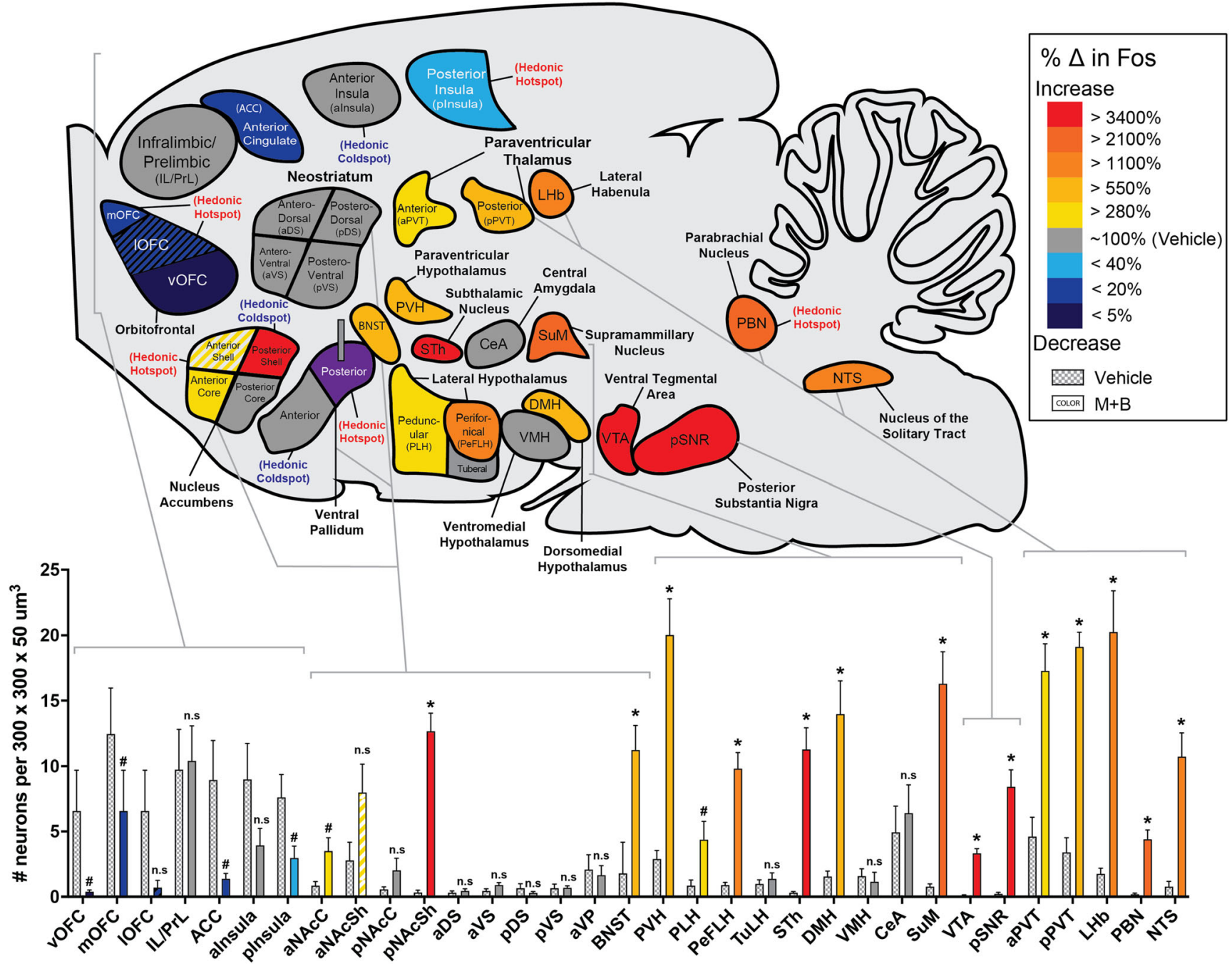

Fig. 6 Whole-brain map of Fos activation changes recruited by excessive "disgust" during pVP inactivation. Sagittal map displays changes in Fos after muscimol + baclofen microinjections, compared with control baseline levels after vehicle microinjections. Colors reflect the significant percentage increase (red, orange, yellow) or decrease (blue, purple) in Fos levels recruited by excessive "disgust" after pVP inactivation compared with vehicle control baselines $(100 \%=$ vehicle baseline, dark gray). Bars show absolute counts of Fos-expressing neurons in each corresponding brain structure. Increases in Fos expression were detected during "disgust" in the following structures: $>280 \%$ in the anterior core of accumbens ( $\mathrm{aNAcC}, \mathrm{p}=0.025$ ), anterior shell of accumbens (aNAcSh, $p=.087$ ), anterior paraventricular nucleus of thalamus (aPVT, $p<.001$ ), and peduncular nucleus of lateral hypothalamus (PLH, $\mathrm{p}=0.039) ;>550 \%$ in the basal nucleus of the stria terminalis (BNST, $p=$ $.002)$, paraventricular nucleus of hypothalamus (PVH, $p<.001)$, and posterior paraventricular nucleus of thalamus (pPVT, $p<.001$ ); $>1,100 \%$ in the perifornical nucleus of hypothalamus (PeFLH, $p<$ .001 ), lateral habenula ( $\mathrm{LHb}, p<.001$ ), and nucleus of the solitary tract (NTS, $p=.001) ;>2,100 \%$ in the supramammilary nucleus (SuM, $p<$

\section{$-2.816, p=.026, d=-1.40$, and a $<20 \%$ trend toward decrease} in the lateral orbitofrontal cortex (1OFC, Levene's $p=.002)$, $t(7.430)=-1.850, p=0.104, d=-0.93$. Similarly, in the insula we found a $<40 \%$ decrease in the posterior insula (pInsula, Levene's $p=0.026), t(11.961)=-2.374, p=$
.001 ) and parabrachial nucleus of the pons (PBN, $p<.001$ ); and $>3,400 \%$ in the posterior shell of accumbens (pNAcSh, $p<.001$ ), subthalamic nucleus (STh, $p<.001$ ), ventral tegmental area (VTA, $p<.001$ ), and posterior substantia nigra (pSNR, $p<.001)$. Decreases in Fos expression were detected during "disgust" in the following cortex regions: $<40 \%$ baseline in the posterior insula cortex (pInsula, $p=.035$ ); $<20 \%$ in the medial orbitofrontal cortex (mOFC, $p=.024)$, and anterior cingulate cortex (ACC, $p=.028) ;<20 \%$ in the lateral orbitofrontal cortex (1OFC, $p=$ .104 ); and $<5 \%$ in the ventral orbitofrontal cortex (vOFC, $p=.026$ ). Muscimol + baclofen microinjection did not cause significant changes in Fos expression in the infralimbic and prelimbic cortex regions of the anterior cingulate lobe (IL/PrL, $p=.871$ ), posterior core of accumbens (pNAcC, $p=.156$ ), anterior portion of ventral pallidum (aVP, $p=.775$ ), anterior insula cortex (aInsula, $p=.126$ ), any of the four quadrants of the neostriatum (aDS, $p=.513$; $\mathrm{aVS}, p=.513 ; \mathrm{pDS}, p=.339 ; \mathrm{pVS}, p=.934$ ), tuberal nucleus of hypothalamus (TuLH, $p=.495$ ), central amygdala (CeA, $p=.627$ ), or ventromedial nucleus of hypothalamus (VMH, $p=$ $.659) . \# p<.05 . * p<.005$. (Color figure online)

.035, $d=-1.13$, which contains another opioid-orexin hedonic hot spot for "liking" enhancement. Medially within the cortex, we found a $<20 \%$ decrease in the anterior cingulate cortex (ACC, Levene's $p=.006$ ), $t(8.331)=-2.654, p=.028, d=-1.25$. 
No significant changes in Fos expression were detected in the infralimbic or prelimbic regions of the anterior cingulate lobe (IL/PrL), $t(16)=0.164, p=.871, d=0.08$, or in the anterior insula cortex (aInsula, Levene's $p=.004), t(11.704)=-1.649, p$ $=.126, d=-0.78$. Subcortically, no changes were detected in the posterior core of the accumbens (pNAcC, Levene's $p=.026$ ), $t(8.758)=1.551, p=.156, d=0.73$, anterior portion of the ventral pallidum $(\mathrm{aVP}), t(12)=-0.292, p=.775, d=-0.15$, any of the four quadrants of the neostriatum, aDS, $t(15)=$ $0.669, p=.513, d=0.34 ;$ aVS, $t(15)=0.669, p=.513, d=$ 0.86 ; pDS, $t(15)=-0.987, p=.339, d=-0.0 .47$; pVS, $t(9.613)=$ $0.085, p=.934, d=0.04$, tuberal nucleus of the hypothalamus, TuLH, $t(16)=0.699, p=.495, d=0.33$, central nucleus of amygdala, CeA, $t(12)=0.499, p=.627, d=0.27$, or ventromedial nucleus of the hypothalamus, $\mathrm{VMH}, t(15)=-0.450, p=$ $.659, d=-0.22$. All distributed Fos changes are shown in Fig. 6.

\section{Discussion}

The ventral pallidum plays a central role in reward-related motivation and emotion (Ahrens, Ferguson, Robinson, \& Aldridge, 2018; Chang, Smedley, Stansfield, Stott, \& Smith, 2017; Mahler et al., 2014; Pardo-Garcia et al., 2019; Rock, Limebeer, Aliasi-Sanai, \& Parker, 2019; Smith et al., 2009; Tachibana \& Hikosaka, 2012). With regard to the hedonic impact of pleasant stimuli, its posterior half ( $\mathrm{pVP}$ ) contains a hedonic hot spot where opioid or orexin stimulation enhances "liking" reactions to sweetness and where neuronal activity tracks the magnitude of positive "liking" (Ho \& Berridge, 2013; Smith \& Berridge, 2005; Tindell et al., 2004; Tindell et al., 2006). The same neurochemical stimulations outside of the pVP hot spot, at sites in anterior VP or other nearby structures, fail to enhance "liking" even if they still enhance "wanting." Conversely, as confirmed here, pVP inactivation by microinjection of a mixture of $\mathrm{GABA}_{\mathrm{A}}$ and $\mathrm{GABA}_{\mathrm{B}}$ receptor agonists (muscimol and baclofen) eliminates normal "liking" reactions, and instead elicits excessive "disgust" even to sweet tastes (Ho \& Berridge, 2014; Shimura et al., 2006).

Human studies also have supported the hypothesis that VP suppression contributes to disgust, and conversely that $\mathrm{pVP}$ activation contributes to positive hedonic impact. For example, a human neuroimaging study found that individual participants who happened to be strongly disgusted by cheese reacted with suppressed VP responses to cheese odors and photos compared with other individuals who enjoyed eating cheese (Royet, Meunier, Torquet, Mouly, \& Jiang, 2016). Conversely, in rats pVP neuronal activation accompanies enhancement of "liking," and in humans pVP activation specifically accompanies positive affective reactions to images of appetizing food (Beaver et al., 2006; Calder et al., 2007). Related to the loss of positive affect produced by pVP inhibition described here, partial damage to a VP-containing region (globus pallidus region) in a human drug addict incurred during a hypoxic overdose produced subsequent anhedonia symptoms and abolished cravings for drugs (Miller et al., 2006).

\section{Mapping "disgust" circuitry: Distant Fos elevations and suppressions caused by pVP inhibition}

Our anatomical analysis of brain activation patterns accompanying pVP inactivation that released excessive "disgust," was based on measuring changes in the transcription and translation of the early immediate c-fos gene into neuronal Fos protein (Herrera \& Robertson, 1996; Sagar, Sharp, \& Curran, 1988). Increases in Fos typically correlated with increases in electrophysiological firing, but in at least a few other cases Fos and firing may diverge. Thus, Fos is an independent form of neuronal activation, particularly reflecting intraneuronal activation of second messenger signaling cascades, and third messenger signals or early immediate genes such as Fos, which in turn act as transcription factors to modulate the expression of other genes that alter neuronal functions.

Here, increases in Fos revealed an anatomical pattern of metabolic activations in subcortical limbic structures, and conversely, simultaneous suppressions in limbic regions of cortex (see Fig. 6). For example, subcortical elevations were found in several structures comprising the basal forebrain around the VP. Inactivation of $\mathrm{pVP}$ produced quintupling of Fos expression in the adjacent bed nucleus of the stria terminalis (BNST) and in a nearby sublenticular part of extended amygdala, both of which are directly posterior to the VP itself. Both BNST and the extended amygdala have been implicated in a number of other aversive motivational states, including stress and anxiety (Davis, Walker, Miles, \& Grillon, 2010; Fox, Oler, Tromp, Fudge, \& Kalin, 2015; Goode \& Maren, 2017; Jennings et al., 2013). Anterior to the VP in the basal forebrain, Fos expression was also elevated by over $3,000 \%$ in the posterior shell of the accumbens (NAc). This posterior subregion of NAc shell contains another opioid "hedonic cold spot," where mu opioid stimulation suppresses "liking" reactions, and where muscimol + baclofen inactivation induces negatively valenced "fearful" reactions and causes excessive "disgust" to sucrose, similarly to pVP inactivation (Faure et al., 2010; Ho \& Berridge, 2014; Reynolds \& Berridge, 2002).

Several cortical regions associated with positive reward function were simultaneously suppressed in Fos expression by pVP-induced "disgust." For example, Fos decreases were produced in the ventromedial subregion of orbitofrontal cortex, which contains another positively valenced hedonic hot spot for opioid or orexin enhancement of "liking" reactions to sucrose taste (Castro \& Berridge, 2017). Fos decreases were also produced in the far posterior region of insula, which similarly contains an opioid-orexin hedonic hot spot (Castro \& Berridge, 2017). 
Beyond limbic structures known to contain hedonic hot spots, several other brain structures showed marked Fos changes during "disgust" induced by $\mathrm{pVP}$ inactivation. In medial hypothalamus, we observed significant Fos elevation in the paraventricular nucleus, dorsomedial nucleus, and supramammilary nucleus. In lateral hypothalamic regions, Fos was elevated in the perifornical nucleus and there was at least a trend toward elevation in the peduncular nucleus. Dorsally in the diencephalon, we observed Fos elevation in the anterior and posterior halves of the paraventricular nucleus of the thalamus, as well as in the subthalamic nucleus and the lateral habenula.

In the midbrain, Fos was elevated in the posterior substantia nigra and in the ventral tegmental area (VTA). We note that both the lateral habenula and VTA receive substantial input projections from VP, and the lateral habenula has been particularly linked to aversive and antireward processes (Geisler \& Zahm, 2005 ; Matsumoto \& Hikosaka, 2007; Proulx, Hikosaka, \& Malinow, 2014; Tandon, Keefe, \& Taha, 2017; Tuesta et al., 2017; Wulff, Tooley, Marconi, \& Creed, 2019). Observed Fos elevations in the VTA may be particularly surprising since it is traditionally known for its role in incentive motivation and reward (Berridge, 2007). However, there are several possibilities that might help explain Fos activation in VTA during "disgust." First, a subpopulation of neurons in VTA has been suggested to produce negatively valenced motivated reactions, including the induction of conditioned place aversion and reduction of reward consumption (Tan et al., 2012; van Zessen, Phillips, Budygin, \& Stuber, 2012), so one possibility is that this aversive VTA subpopulation participates in generating "disgust." Second, VTA activation might alternatively reflect nonvalenced general arousal or sensorimotor activation during "disgust" reactions. Third, if an opponent-process mechanism were involved (Solomon \& Corbitt, 1974), then VTA activation could even reflect activation of incentive circuitry as a partial compensatory response or bprocess, triggered by the stronger aversive a-process of excessive "disgust" induced by pVP inhibition. Future studies may help to choose amongst these or additional explanations for the role of VTA activation in "disgust."

In the hindbrain, Fos expression was elevated in the nucleus of the solitary tract (NTS) of the medulla and in the parabrachial nucleus of the pons (PBN). These two brain stem nuclei are, respectively, the first and second sensory relay nuclei for ascending gustatory and visceral sensory signals in the brain (Norgren \& Leonard, 1971). Further, the PBN in particular has been suggested to be an important "somatic marker" mechanism of bodily feelings in emotions (Damasio, 1999), as well as the source of negatively valenced "alarm" signals regarding aversive tastes and painful stimuli (Palmiter, 2018).

Overall, in comparing cortical and subcortical changes in Fos during excessive "disgust," it is striking that only Fos suppressions, and not any elevations, were detected in the cortex regions analyzed here, whereas conversely, only Fos elevations, and not suppressions, were found in subcortical structures (see Fig. 6). This cortical/subcortical divergence may suggest that excessive sensory disgust is generated primarily by activation in subcortical limbic circuitry, released into activity by neighboring $\mathrm{pVP}$ inhibition. On the other hand, limbic cortical regions implicated in hedonic reactions become relatively suppressed, perhaps representing loss of positive "liking," and, via its inhibition, also contributing to negatively valenced release.

\section{Release of "disgust" by related brain manipulations}

Beyond neural inhibitions strictly limited to $\mathrm{pVP}$, we note that similar excessive negative "disgust" is produced by larger lateral hypothalamic lesions, which typically also involved damage in the adjacent $p$ VP (Schallert \& Whishaw, 1978; Stellar, Brooks, \& Mills, 1979; Teitelbaum \& Epstein, 1962). Other related negative-affective release phenomena that might have also involved pVP impairment include classic "thalamic rage" or "thalamic disgust," produced by a surgical procedure called the "thalamic preparation" that entails removal of all cortical and subcortical brain structures anterior to thalamus (i.e., telencephalon removal; Bard, 1934; Cannon, 1927; Grill \& Norgren, 1978a), and similarly by purely ventral forebrain ablations that extend posteriorly from the olfactory tubercle to invade the ventral pallidum (Fulton \& Ingram, 1929; Spiegel, Miller, \& Oppenheimer, 1940). By contrast, excessive negative-valenced "disgust" or "rage" reactions are not produced by lower decerebration transections that leave intact only the midbrain and hindbrain (Grill \& Norgren, 1978b; Miller \& Sherrington, 1915). This difference suggests that prime candidates for release of negative affective reactions might especially be in the hypothalamus, ventral thalamus, or in portions of the ventral telencephalon that are adjacent and posterior to the ventral pallidum but anterior to the midbrain, such as BNST or adjacent sublenticular parts of the extended amygdala. All of those structures were activated in excessive "disgust" here, including specific hypothalamic nuclei, the lateral habenula, BNST, and the sublenticular extended amygdala.

\section{Excessive "disgust" as a release phenomenon after pVP inhibition}

Excessive "disgust" was achieved here via induced hyperpolarization of neurons in the pVP, caused by neurochemical binding of muscimol + baclofen to neuronal GABA receptors. GABA receptor activation opens chloride ion channels, allowing negatively charged chloride ions to enter the neuron and create inhibitory postsynaptic potentials that suppress neuronal activity. Since many pVP neurons release GABA themselves, $p V P$ neuronal inhibition induces disinhibition of neurons in projection targets directly downstream, allowing those targets to become neurbiologically excited by shutting 
off their VP GABAergic inhibition. These targets include several of the subcortical structures described to have elevated Fos expression above. Such disinhibited structures, showing neurobiological excitation, may serve as the best candidates for actual generators of excessive "disgust" reactions.

This would mean that excessive "disgust" triggered by pVP inhibition is best viewed as a release phenomenon (Ho \& Berridge, 2014). That is, the pVP inhibition by muscimol + baclofen microinjection causes sudden loss of neural circuitry in the pVP hedonic hot spot that is able to generate positively valenced "liking" reactions, releasing the unopposed expression of excessive negative affective reactions generated by circuitry elsewhere, such as in the structures described as activated above. The concept of a neural release phenomenon may be owed to the 19th-century British neurologist John Hughlings Jackson (1958/ 1879), who stated that a neural lesion or loss causes a "negative mental condition" that permits "positive mental symptoms" to emerge. Applied here, the "negative mental condition" would refer to a loss of hedonic "liking" function. Jackson described such release phenomena in the context of human patients with brain damage: "We must never speak of destructive lesions causing positive symptoms. It is erroneous, I submit to say that any sort of disease causes elaborate positive mental symptoms, illusions, hallucinations, etc." Instead, "it causes a negative mental condition, the elaborate positive mental symptoms are permitted' (p. 192, italics in original).

Regarding "liking" versus "disgust," the "negative mental condition" referred to by Jackson would mean a loss of hedonic "liking" function as observed in the present study. By "positive mental symptoms" Jackson meant not positively valenced affect, but instead the emergence of a new and active mental psychological phenomenon, such as the expression of excessive "disgust" reactions here, that is consequently released into overexcitation by sudden loss of inhibition or opposition. Similar release interpretations might be applied to all the other neural manipulations that produce intense "disgust" or "rage" mentioned above (Bard, 1934; Cannon, 1927; Fulton \& Ingram, 1929; Grill \& Norgren, 1978a; Schallert \& Whishaw, 1978; Spiegel et al., 1940; Stellar et al., 1979; Teitelbaum \& Epstein, 1962). Applied to pVP and "disgust," a release interpretation means that $\mathrm{pVP}$ circuitry does not generate the excessive "disgust" reaction itself, but rather serves as a mechanism for positively valenced "liking" reactions that normally oppose or suppress "disgust"-generating circuitry elsewhere in the brain. The brain structures identified above as showing increases in Fos expression are excellent candidates for that "disgust"-generating circuitry.

\section{Sensorimotor restructuring of the hierarchy of "disgust" reactions}

Under normal conditions, aversive paw treading is relatively rare compared to other "disgust" reactions evoked by bitter tastes, such as gapes, headshakes, chin rubs or forelimb flails (Berridge \& Fentress, 1986). However, previous studies showed that excitotoxin lesions of the VP and nearby globus pallidus cause a restructuring of the sensorimotor hierarchy of "disgust" reactions. Especially potentiated is paw treading behavior to oral quinine infusion, or to sucrose infusions during the days to weeks after pVP lesions when sucrose elicits "disgust"; in both cases, such treading becomes exaggerated or hyperkinetic, and can persist for several minutes after the oral infusion ends (Berridge \& Cromwell, 1990; Cromwell \& Berridge, 1993). In our study, hyperkinetic treading as a "disgust" reaction was potentiated by temporary pharmacologic $\mathrm{pVP}$ inactivations, and once induced, additionally appeared subsequently as a Pavlovian conditioned reaction elicited by environment associated with previous oral taste infusions. "Disgust" occurring as a Pavlovian conditioned response when the rat is placed in a chamber that triggers a learned association with sucrose infusions is similar to prior reports of contextually elicited "disgust" behavior, in which aversive gape reactions were elicited by environment in which a previous sweet taste experience had been associated with the nausea induced by a lithium chloride injection (Limebeer, Hall, \& Parker, 2006; Rock, Limebeer, \& Parker, 2014). Interestingly, these investigators went on to report that conditioned gaping reactions could be diminished by microinjections into VP of an inhibitor of fatty acid amide hydrolase (FAAH; Rock et al., 2019). FAAH degrades the endocannabinoid neurotransmitter anandamide and related compounds, though Rock and colleagues also found evidence that conditioned "disgust" was suppressed primarily in VP through a PPAR $\alpha$ receptor mediated mechanism, involved in lipid metabolism and potentially in other neuronal functions (Rock et al., 2019). It would be of interest for future studies to examine the roles of FAAH, or of VP neurons with PPAR $\alpha$ receptors in particular, in excessive "disgust" released by $\mathrm{pVP}$ inactivation.

As a potential neural motor-related mechanism corresponding to the sensorimotor change that particularly promoted hyperkinetic treading among "disgust" reactions, we observed strong Fos expression in structures such as the subthalamic nucleus and posterior substantia nigra pars reticularis (pSNR), which are implicated in several clinical movement disorders in humans (Limousin et al., 1995; Périer, Tremblay, Féger, \& Hirsch, 2002; Salin, Manrique, Forni, \& Kerkerian-Le Goff, 2002), and in "abnormal limb movements" in rats (Périer et al., 2002). These structures are anatomically interconnected with each other and with the VP (Haber, Groenewegen, Grove, \& Nauta, 1985; Parent \& Hazrati, 1995). The VP sends GABAergic input to the medial subthalamic nucleus (Haber et al., 1985; Parent \& Hazrati, 1995), which sends glutamatergic projections to the SNR, so it is possible that $\mathrm{pVP}$ inactivation may disinhibit excitatory subthalamic projections to SNR. This could result in pSNR excitation, potentially helping to generate motor dysfunctions such as the hyperkinetic treading observed here. The subthalamic nucleus is also implicated in reward- 
related behavior and incentive salience (Baunez, Amalric, \& Robbins, 2002; Rossi, Fan, Barter, \& Yin, 2013; Serranová et al., 2011; Uslaner \& Robinson, 2006), and conceivably also plays a role in potentiating hyperkinetic treading specifically during affective "disgust" states.

\section{Impaired appetitive motivation and induction of avoidance}

Circuitry released by pVP inhibition might also be involved in producing negative motivational reactions to food or a social partner. For example, pVP inactivation eliminated the appetitive motivation of "wanting" to eat palatable food in the voluntary intake test, which is similar to aphagia caused by excitotoxic lesions of pVP (Berridge \& Cromwell, 1990; Cromwell \& Berridge, 1993), as well as by large electrolytic lesions of lateral hypothalamus in early studies (Morgane, 1961; Schallert \& Whishaw, 1978; Stellar et al., 1979; Teitelbaum \& Epstein, 1962), which also probably invaded the pVP (Castro, Cole, \& Berridge, 2015).

Regarding social motivation, $\mathrm{pVP}$ inactivation also suppressed positive social interactions with a social partner (investigatory sniffing, following, and physical contacts), and instead induced either social passivity or even actively negative social avoidance and escape when the partner approached. This appears related to social avoidance reported to be produced in rats recovering from large lateral hypothalamic lesions that probably also damaged the pVP (Schallert \& Whishaw, 1978). We note that bilateral muscimol microinjection in the VP has similarly been reported to disrupt maternal behavior (Numan et al., 2005).

\section{Conclusion}

In summary, our results show that pharmacological GABAergic inactivation of $\mathrm{pVP}$, which converts sweetness "liking" to excessive "disgust," recruits changes in an extensive brain limbic network that includes candidate "disgust" generators. These included excitation of a number of subcortical limbic structures, as well as simultaneous suppression of several cortical regions that might be related to positive "liking." Such neural circuitry changes may be involved in causing the released generation of excessive negative "disgust" reactions after pVP inhibition. These results may therefore shed light on the neurobiological basis of intense sensory disgust, and may also be relevant to understanding clinical affective disorders characterized by either anhedonia and/or excessive dysphoria.

Acknowledgements This work was supported by National Institutes of Health grants MH063649 and DA015188 (to K.C.B.). K.R.U. was supported by National Institutes of Health training grant DC00011. We would like to thank Cody Schember, Josh Goldman, Erin Grant, Tyler Allerton, and Nicholas Rabah for their assistance in conducting these experiments.
Open Practices Statement None of the data or materials for the experiments reported here is available, and none of the experiments was preregistered.

\section{References}

Ahrens, A. M., Ferguson, L. M., Robinson, T. E., \& Aldridge, J. W. (2018). Dynamic encoding of incentive salience in the ventral pallidum: Dependence on the form of the reward cue. eNeuro, 5(2). doi:https://doi.org/10.1523/ENEURO.0328-17.2018

Arrondo, G., Murray, G. K., Hill, E., Szalma, B., Yathiraj, K., Denman, C., \& Dudas, R. B. (2015). Hedonic and disgust taste perception in borderline personality disorder and depression. The British Journal of Psychiatry, 207, 79-80.

Bard, P. (1934). On emotional expression after decortication with some remarks on certain theoretical views. Psychological Review, 41, 309-329.

Baunez, C., Amalric, M., \& Robbins, T. W. (2002). Enhanced foodrelated motivation after bilateral lesions of the subthalamic nucleus. Journal of Neuroscience, 22, 562-568.

Beaver, J. D., Lawrence, A. D., Van Ditzhuijzen, J., Davis, M. H., Woods, A., \& Calder, A. J. (2006). Individual differences in reward drive predict neural responses to images of food. Journal of Neuroscience, 26, 5160-5166.

Berridge, K. C. (2000). Measuring hedonic impact in animals and infants: Microstructure of affective taste reactivity patterns. Neuroscience and Biobehavioral Reviews, 24, 173-198

Berridge, K. C. (2007). The debate over dopamine's role in reward: The case for incentive salience. Psychopharmacology (Berl), 191, 391431.

Berridge, K. C. (2018). Evolving concepts of emotion and motivation. Frontiers in Psychology, 9, 1-20.

Berridge, K. C., \& Cromwell, H. C. (1990). Motivational-sensorimotor interaction controls aphagia and exaggerated treading after striatopallidal lesions. Behavioral Neuroscience, 104, 778-795.

Berridge, K. C., \& Fentress, J. C. (1986). Contextual control of trigeminal sensorimotor function. Journal of Neuroscience, 6, 325-330.

Berridge, K. C., \& Kringelbach, M. L. (2015). Pleasure systems in the brain. Neuron, 86, 646-664.

Calder, A. J., Beaver, J. D., Davis, M. H., Van Ditzhuijzen, J., Keane, J., \& Lawrence, A. D. (2007). Disgust sensitivity predicts the insula and pallidal response to pictures of disgusting foods. European Journal of Neuroscience, 25, 3422-3428.

Cannon, W. B. (1927). The James-Lange theory of emotions: A critical examination and an alternative theory. The American Journal of Psychology, 39, 106-124.

Castro, D. C., \& Berridge, K. C. (2017). Opioid and orexin hedonic hot spots in rat orbitofrontal cortex and insula. Proceedings of the National Academy of Sciences, 114, 9125-9134.

Castro, D. C., Cole, S. L., \& Berridge, K. C. (2015). Lateral hypothalamus, nucleus accumbens, and ventral pallidum roles in eating and hunger: Interactions between homeostatic and reward circuitry. Frontiers in Systems Neuroscience, 9. doi:https://doi.org/10.3389/ fnsys.2015.00090

Chang, S. E., Smedley, E. B., Stansfield, K. J., Stott, J. J., \& Smith, K. S. (2017). Optogenetic inhibition of ventral pallidum neurons impairs context-driven salt seeking. The Journal of Neuroscience, 37, 5670 5680 .

Cromwell, H. C., \& Berridge, K. C. (1993). Where does damage lead to enhanced food aversion: The ventral pallidum/substantia innominata or lateral hypothalamus? Brain Research, 624, 1-10.

Cromwell, H. C., \& Berridge, K. C. (1994). Mapping of globus pallidus and ventral pallidum lesions that produce hyperkinetic treading. Brain Research, 668, 16-29. 
Darwin, C. (1872). The expression of the emotions in man and animals (1998 ed., revised and with commentary by P. Ekman). Oxford, UK: HarperCollins.

Damasio, A. R. (1999). The feeling of what happens: Body and emotion in the making of consciousness. New York, NY: Harcourt Brace.

Davey, G. C. L. (2011). Disgust: The disease-avoidance emotion and its dysfunctions. Philosophical Transactions of the Royal Society B: Biological Sciences, 366, 3453-3465.

Davis, M., Walker, D. L., Miles, L., \& Grillon, C. (2010). Phasic vs sustained fear in rats and humans: Role of the extended amygdala in fear vs anxiety. Neuropsychopharmacology, 35, 105-135.

Faure, A., Richard, J. M., \& Berridge, K. C. (2010). Desire and dread from the nucleus accumbens: Cortical glutamate and subcortical GABA differentially generate motivation and hedonic impact in the rat. PLOS ONE, 5, e11223.

Fluharty, S. J., \& Grill, H. J. (1981). Taste reactivity of lateral hypothalamic lesioned rats: Effects of deprivation and tube feeding. Neuroscience Abstracts, 7, 29.

Fox, A. S., Oler, J. A., Tromp, D. P. M., Fudge, J. L., \& Kalin, N. H. (2015). Extending the amygdala in theories of threat processing. Trends in Neurosciences, 38, 319-329.

Fulton, J. F., \& Ingram, F. D. (1929). Emotional disturbances following experimental lesions of the base of the brain (pre-chiasmal). American Journal of Physiology, 90, 353.

Geisler, S., \& Zahm, D. S. (2005). Afferents of the ventral tegmental area in the rat-anatomical substratum for integrative functions. Journal of Comparative Neurology, 490, 270-294.

Goode, T. D., \& Maren, S. (2017). Role of the bed nucleus of the stria terminalis in aversive learning and memory. Learning and Memory, $24,480-491$.

Grill, H. J., \& Norgren, R. (1978a). The taste reactivity test: I. Mimetic responses to gustatory stimuli in neurologically normal rats. Brain Research, 143, 263-279.

Grill, H. J., \& Norgren, R. (1978b). The taste reactivity test: II. Mimetic responses to gustatory stimuli in chronic thalamic and chronic decerebrate rats. Brain Research, 143, 281-297.

Haber, S. N., Groenewegen, H. J., Grove, E. A., \& Nauta, W. J. H. (1985). Efferent connections of the ventral pallidum: Evidence of a dual striato pallidofugal pathway. Journal of Comparative Neurology, 235, 322-335.

Herrera, D. G., \& Robertson, H. A. (1996). Activation of c-fos in the brain. Progress in Neurobiology, 50, 83-107.

Ho, C. Y., \& Berridge, K. C. (2013). An orexin hot spot in ventral pallidum amplifies hedonic "liking" for sweetness. Neuropsychopharmacology, 39, 1655-1664.

Ho, C. Y., \& Berridge, K. C. (2014). Excessive disgust caused by brain lesions or temporary inactivations: Mapping hot spots of the nucleus accumbens and ventral pallidum. European Journal of Neuroscience, 40, 3556-3572.

Hughlings Jackson, J. (1958). Selected writings of John Hughlings Jackson. The American Journal of the Medical Sciences. (Original work published 1879)

Jennings, J. H., Sparta D. L., Stamatakis, A. M., Ung, R. L., Pleil, K. E., Kash, T. L., \& Stuber G. D (2013). Distinct extended amygdala circuits for divergent motivational states. Nature, 496, 224-228

Keltner, D., \& Ekman, P. (2000). Facial expression of emotion. In M. Lewis \& J. Haviland-Jones (Eds.), Handbook of emotions (2nd ed., pp. 236-249). New York, NY: Guilford Press.

Limebeer, C. L., Hall, G., \& Parker, L. A. (2006). Exposure to a lithiumpaired context elicits gaping in rats: A model of anticipatory nausea. Physiology \& Behavior, 88, 398-403,

Limousin, P., Pollak, P., Benazzouz, A., Hoffmann, D., Le Bas, J. F., Perret, J. E., ... Broussolle, E. (1995). Effect on parkinsonian signs and symptoms of bilateral subthalamic nucleus stimulation. The Lancet, 345, 91-95.
Mahler, S. V., Vazey, E. M., Beckley, J. T., Keistler, C. R., Mcglinchey, E. M., Kaufling, J., ... Aston-Jones, G. (2014). Designer receptors show role for ventral pallidum input to ventral tegmental area in cocaine seeking. Nature Neuroscience, 17, 577-585.

Matsumoto, M., \& Hikosaka, O. (2007). Lateral habenula as a source of negative reward signals in dopamine neurons. Nature, 447(7148), 1111-1115.

Miller, F. R., \& Sherrington, C. S. (1915). Some observations on the bucco-pharyngeal stage of reflex deglutition in the cat. Quarterly Journal of Experimental Physiology, 9, 147-186.

Miller, J. M., Vorel, S. R., Tranguch, A. J., Kenny, E. T., Mazzoni, P., Van Gorp, W. G., \& Kleber, H. D. (2006). Anhedonia after a selective bilateral lesion of the globus pallidus. American Journal of Psychiatry, 163, 786-788.

Morgane, P. J. (1961). Distinct "feeding" and "hunger motivating" systems in the lateral hypothalamus of the rat. Science, 133, 887-888.

Norgren, R., \& Leonard, C. M. (1971). Taste pathways in rat brainstem. Science, 173, 1136-1139.

Numan, M., Numan, M. J., Schwarz, J. M., Neuner, C. M., Flood, T. F., \& Smith, C. D. (2005). Medial preoptic area interactions with the nucleus accumbens-ventral pallidum circuit and maternal behavior in rats. Behavioural Brain Research, 158, 53-68.

Palmiter, R. D. (2018). The parabrachial nucleus: CGRP neurons function as a general alarm. Trends in Neurosciences, 41, 280-293.

Pardo-Garcia, T. R., Garcia-Keller, C., Penaloza, T., Richie, C. T., Pickel J., Hope, B. T., ... Heinsbroek J. A. (2019). Ventral pallidum is the primary target for accumbens D1 projections driving cocaine seeking. The Journal of Neuroscience, 39, 2041-2051.

Parent, A., \& Hazrati, L. N. (1995). Functional anatomy of the basal ganglia: II. The place of subthalamic nucleus and external pallidium in basal ganglia circuitry. Brain Research Reviews, 20, 128-154.

Paxinos, G., \& Watson, C. (2007). The rat brain in stereotaxic coordinates (6th ed.). New York, NY: Elsevier Academic Press.

Peciña, S., \& Berridge, K. C. (2005). Hedonic hot spot in nucleus accumbens shell: Where do mu-opioids cause increased hedonic impact of sweetness? Journal of Neuroscience, 25, 11777-11786.

Périer, C., Tremblay, L., Féger, J., \& Hirsch, E. C. (2002). Behavioral consequences of bicuculline injection in the subthalamic nucleus and the zona incerta in rat. Journal of Neuroscience, 22, 8711-8719.

Pfaffman, C., Norgren, R., \& Grill, H. J. (1977). Sensory affect and motivation. Annals of the New York Academy of Sciences, 290, $18-34$.

Proulx, C. D., Hikosaka, O., \& Malinow, R. (2014). Reward processing by the lateral habenula in normal and depressive behaviors. Nature Neuroscience, 17(9), 1146-1152.

Reynolds, S. M., \& Berridge, K. C. (2001). Fear and feeding in the nucleus accumbens shell: Rostrocaudal segregation of GABAelicited defensive behavior versus eating behavior. Journal of Neuroscience, 21, 3261-3270.

Reynolds, S. M., \& Berridge, K. C. (2002). Positive and negative motivation in nucleus accumbens shell: Bivalent rostrocaudal gradients for GABA-elicited eating, taste "liking"/"disliking" reactions, place preference/avoidance, and fear. Journal of Neuroscience, 22, 73087320.

Richard, J. M., \& Berridge, K. C. (2011). Nucleus accumbens dopamine/ glutamate interaction switches modes to generate desire versus dread: $\mathrm{D}(1)$ alone for appetitive eating but $\mathrm{D}(1)$ and $\mathrm{D}(2)$ together for fear. Journal of Neuroscience, 7(31), 12866-12879.

Rock, E. M., Limebeer, C. L., Aliasi-Sanai, L., \& Parker, L. A. (2019). The ventral pallidum as a critical region regulating fatty acid amide hydrolase inhibition of nausea-induced conditioned gaping in male Sprague-Dawley rats. Neuropharmacology, 155, 142-149

Rock, E. M., Limebeer, C. L., \& Parker, L. A. (2014). Anticipatory nausea in animal models: A review of potential novel therapeutic treatments. Experimental Brain Research, 232, 2511-2534 
Rossi, M. A., Fan, D., Barter, J. W., \& Yin, H. H. (2013). Bidirectional modulation of substantia nigra activity by motivational state. PLOS ONE, 8, e71598.

Royet, J.-P., Meunier, D., Torquet, N., Mouly, A.-M., \& Jiang, T. (2016). The neural bases of disgust for cheese: An fMRI study. Frontiers in Human Neuroscience, 10, 511.

Rozin, P., Haidt, J., \& McCauley, C. R. (2008). Disgust. In M. Lewis \& J. Haviland (Eds.), Handbook of emotions (3rd ed., pp. 757-776). New York, NY: Gilford Press.

Sagar, S. M., Sharp, F. R., \& Curran, T. (1988) Expression of c-Fos protein in brain: Metabolic mapping at the cellular level. Science, 240, 1328-1331.

Salin, P., Manrique, C., Forni, C., \& Kerkerian-Le Goff, L. (2002). Highfrequency stimulation of the subthalamic nucleus selectively reverses dopamine denervation-induced cellular defects in the output structures of the basal ganglia in the rat. Journal of Neuroscience, 22, 5137-5148.

Schallert, T., \& Whishaw, I. Q. (1978). Two types of aphagia and two types of sensorimotor impairment after lateral hypothalamic lesions: Observations in normal weight, dieted, and fattened rats. Journal of Comparative and Physiological Psychology, 92, 720-741.

Serranová, T., Jech, R., Dušek, P., Sieger, T., Růžička, F., Urgošík, D., \& Růžička, E. (2011). Subthalamic nucleus stimulation affects incentive salience attribution in Parkinson's disease. Movement Disorders, 26, 2260-2266.

Shimura, T., Imaoka, H., \& Yamamoto, T. (2006). Neurochemical modulation of ingestive behavior in the ventral pallidum. European Journal of Neuroscience, 23, 1596-1604.

Smith, K. S., \& Berridge, K. C. (2005). The ventral pallidum and hedonic reward: Neurochemical maps of sucrose "liking" and food intake. Journal of Neuroscience, 25, 8637-8649.

Smith, K. S., \& Berridge, K. C. (2007). Opioid limbic circuit for reward: Interaction between hedonic hot spots of nucleus accumbens and ventral pallidum. Journal of Neuroscience, 27, 1594-1605.

Smith, K. S., Berridge, K. C., \& Aldridge, J. W. (2011). Disentangling pleasure from incentive salience and learning signals in brain reward circuitry. Proceedings of the National Academy of Sciences, 108, E255-E264.

Smith, K. S., Tindell, A. J., Aldridge, J. W., \& Berridge, K. C. (2009). Ventral pallidum roles in reward and motivation. Behavioural Brain Research, 196, 155-167.

Solomon, R. L., \& Corbit, J. D. (1974). An opponent-process theory of motivation: I. Temporal dynamics of affect. Psychological Review, 81, 119-145.

Spiegel, E. A., Miller, H. R., \& Oppenheimer, M. J. (1940). Forebrain and rage reactions. Journal of Neurophysiology, 3(6), 538-548.

Steiner, J. E. (1973). The gustofacial response: Observation on normal and anencephalic newborn infants. Symposium of Oral Sensation and Perception, 4, 254-278.
Steiner, J. E., Glaser, D., Hawilo, M. E., \& Berridge, K. C. (2001). Comparativeexpression of hedonic impact: Affective reactions to taste by human infants and other primates. Neuroscience and Biobehavioral Reviews, 25, 53-74.

Stellar, J. R., Brooks, F. H., \& Mills, L. E. (1979). Approach and withdrawal analysis of the effects of hypothalamic stimulation and lesions in rats. Journal of Comparative and Physiological Psychology, 93, 446-466.

Swanson, L. W. (2004). Brain maps: Structure of the rat brain (2nd ed.). New York, NY: Elsevier.

Tachibana, Y., \& Hikosaka, O. (2012). The primate ventral pallidum encodes expected reward value and regulates motor action. Neuron, 76, 826-837.

Tan, K. R., Yvon, C., Turiault, M., Mirzabekov, J. J., Doehner, J., Labouebe, G., ... Luscher, C. (2012). GABA neurons of the VTA drive conditioned place aversion. Neuron, 73, 1173-1183.

Tandon, S., Keefe, K. A., \& Taha, S. A. (2017). Excitation of lateral habenula neurons as a neural mechanism underlying ethanolinduced conditioned taste aversion. Journal of Physiology, 595(4), $1393-1412$.

Teitelbaum, P., \& Epstein, A. N. (1962). The lateral hypothalamic syndrome: Recovery of feeding and drinking after lateral hypothalamic lesions. Psychological Review, 69, 74-90.

Tindell, A. J., Berridge, K. A., \& Aldridge, J. W. (2004). Ventral pallidal representation of pavlovian cues and reward: Population and rate codes. Journal of Neuroscience, 24(5), 1058-1069.

Tindell, A. J., Smith, K. S., Peciña, S., Berridge, K. C., \& Aldridge, J. W. (2006). Ventral pallidum firing codes hedonic reward: When a bad taste turns good. Journal of Neurophysiology, 95, 2399-2409.

Tuesta, L. M., Chen, Z., Duncan, A., Fowler, C. D., Ishikawa, M., Lee, B. R., ... Kenny, P. J. (2017). GLP-1 acts on habenular avoidance circuits to control nicotine intake. Nature Neuroscience, 20, 708716.

Uslaner, J. M., \& Robinson, T. E. (2006). Subthalamic nucleus lesions increase impulsive action and decrease impulsive choiceMediation by enhanced incentive motivation? European Journal of Neuroscience, 24, 2345-2354.

van Zessen, R., Phillips, J. L., Budygin, E. A., \& Stuber, G. D. (2012). Activation of VTA GABA neurons disrupts reward consumption. Neuron, 73, 1184-1194.

Wulff, A. B., Tooley, J., Marconi, L. J., \& Creed, M. C. (2019). Ventral pallidal modulation of aversion processing. Brain Research, 1713, $62-69$.

Publisher's note Springer Nature remains neutral with regard to jurisdictional claims in published maps and institutional affiliations. 